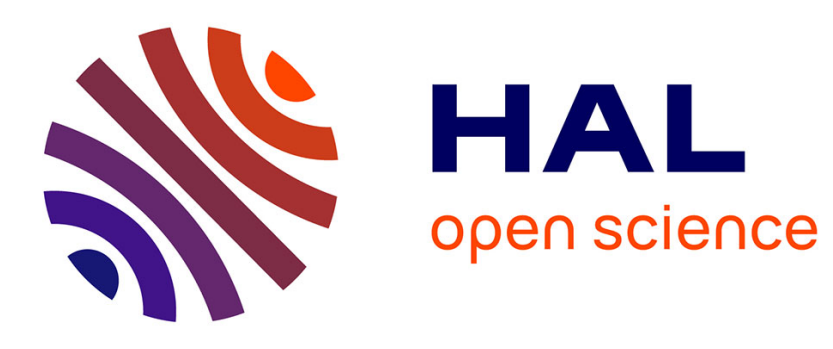

\title{
An algebraic approach to discrete dilations. Application to discrete wavelet transforms
}

\author{
Jean-Pierre Antoine, Yebeni B. Kouagou, Dominique Lambert, Bruno
}

Torrésani

\section{- To cite this version: \\ Jean-Pierre Antoine, Yebeni B. Kouagou, Dominique Lambert, Bruno Torrésani. An algebraic ap- proach to discrete dilations. Application to discrete wavelet transforms. Journal of Fourier Analysis and Applications, 2000, 6 (2), pp.113-141. 10.1007/BF02510656 . hal-00814185}

\author{
HAL Id: hal-00814185 \\ https://hal.science/hal-00814185
}

Submitted on 16 Apr 2013

HAL is a multi-disciplinary open access archive for the deposit and dissemination of scientific research documents, whether they are published or not. The documents may come from teaching and research institutions in France or abroad, or from public or private research centers.
L'archive ouverte pluridisciplinaire HAL, est destinée au dépôt et à la diffusion de documents scientifiques de niveau recherche, publiés ou non, émanant des établissements d'enseignement et de recherche français ou étrangers, des laboratoires publics ou privés. 


\title{
An algebraic approach to discrete dilations. Application to discrete wavelet transforms
}

\author{
J.- P. Antoine Y. B. Kouagou $†$ \\ Institut de Physique Théorique, \\ Université Catholique de Louvain, \\ B-1348 Louvain-la-Neuve, Belgium. \\ D. LAMBert \\ Faculté des Sciences, FUNDP, \\ B-5000 Namur, Belgium. \\ and \\ B. Torrésani \\ Laboratoire d'Analyse, Topologie et Probabilités, \\ CMI, Université de Provence, 32 Avenue F. Joliot-Curie, \\ 13453 Marseille, France.
}

1998

\begin{abstract}
We investigate the connections between continuous and discrete wavelet transforms on the basis of algebraic arguments. The discrete approach is formulated abstractly in terms of the action of a semidirect product $\mathcal{A} \times \Gamma$ on $\ell^{2}(\Gamma)$, with $\Gamma$ a lattice and $\mathcal{A}$ an abelian semigroup acting on $\Gamma$. We show that several such actions may be considered, and investigate those which may be written as deformations of the canonical one. The corresponding deformed dilations (the pseudodilations) turn out to be characterized by compatibility relations of a cohomological nature. The connection with multiresolution wavelet analysis is based on families of pseudodilations of a different type.
\end{abstract}

UCL-IPT-98-17

November1998

† Permanent address: Institut de Mathématiques et de Sciences Physiques, BP-613 Porto-Novo, Bénin, West Africa.

‡ Former affiliation: Centre de Physique Théorique, CNRS Luminy, Case 907, F-13288 Marseille Cedex 


\section{Contents}

1 Introduction $\quad 3$

2 Pseudodilations and associated compatible filters 4

2.1 Background, notations ....................... 4

2.2 Principal pseudodilations . . . . . . . . . . . . . . . . . . 6

2.3 Associated pseudodilation . . . . . . . . . . . . . . . . . . . . . . . . . . . . . . .

2.4 Adjoint pseudodilations and inversion . . . . . . . . . . . . . . . 11

2.5 Compatible filters in the case $\mathcal{G}=\mathbb{R}, \mathcal{A} \subset \mathbb{Z}_{+} \ldots \ldots \ldots 13$

3 Cohomological interpretation $\quad 15$

3.1 Generalities . . . . . . . . . . . . . . . . . . 15

3.2 The module of $n$-cochains . . . . . . . . . . . . . . . . 16

3.3 Differentials and cohomology groups . . . . . . . . . . . . . . . . 17

3.4 Cohomological solution of the pseudodilation filters equations . . . . . . . . . 19

4 Discretized wavelet transform and generalized pyramid algorithms 21

4.1 Action of the filters on functions . . . . . . . . . . . . . . . . . . . 21

4.2 The case $\mathcal{G}=\mathbb{R}, \Gamma=\mathbb{Z}$ and $\mathcal{A} \subset \mathbb{Z}_{+} \ldots \ldots \ldots \ldots \ldots$. . . . . . . . . . . . . . . . . . . . . . . . . . 22

4.3 Discrete wavelet transforms . . . . . . . . . . . . . . . . . 25

5 Conclusion $\quad 28$

A Notions of harmonic analysis on locally compact abelian groups 31

$\begin{array}{ll}\text { B Basic operations } & 33\end{array}$

$\begin{array}{ll}\text { C Sampling } & 33\end{array}$ 


\section{Introduction}

Wavelet analysis and related methods have received considerable attention during the past ten years, both in theoretical and in applied sciences. In our opinion, the main reason of such a success is, besides the relative simplicity of the tool, its extreme computational efficiency. The latter is deeply related to the so-called multiresolution structure associated to wavelets, responsible for the existence of fast and accurate algorithms for computing wavelet transforms (the so-called pyramid algorithms). The interested reader may refer to [27, 28] for a detailed discussion of these algorithmic aspects. The main point here is that the wavelet decompositions considered in such an approach are intrinsically discrete decompositions.

Besides such an algorithmic point of view, wavelets have also received a great attention in the mathematical physics community [26], especially because of their deep relation with coherent states (see [1] for a recent review). In that approach, the emphasis is put on the symmetries, in particular the fact that the wavelet transform, i.e., the transform which maps a function into the coefficients of its decomposition with respect to a family of wavelets, is covariant with respect to a group action: the affine group of the real line in the case of usual wavelets, or bigger groups in more general situations. In all cases, the groups under consideration are continuous groups. The use of such symmetries is at least implicit in many applications, for example in signal analysis (see for example the discussions in $[7,22]$ ).

Remarkably enough, little is known about the connections between the two above mentioned approaches (see nevertheless [15] for a formulation of Gabor transform on locally compact abelian groups), at least as far as algebraic arguments are concerned. In particular, the pyramid algorithms, which are obviously attached to rigid algebraic structures, do not seem to have been considered as such (see nevertheless the discussion of embedding of sampling spaces in [18]). The goal of this paper is to start developing such a point of view, and to make a connection with the group theoretical approach. Part of the results presented here are not new from the wavelet point of view, at least in the case of the usual one-dimensional multiresolution wavelet theory. However, we believe that our construction gives a different perspective on the theory, for it makes the connection with different fields (e.g. homological algebra), which may prove useful for concrete problems (for example the complete classification of perfect reconstruction quadrature mirror filters). It also poses the problem of generalizing multiresolution analyses, in terms close to those developed by $[4,11]$.

Making the connection between the group theoretical approach and the multiresolution approach to wavelets is not an easy task, since no affine group action is available in the discrete case (unless one limits the analysis to discrete fields such as $\mathbb{Z} / p \mathbb{Z}$, with $p$ a prime number, as was done in $[10,20])$. In this paper, we investigate situations where a semigroup $\mathcal{A}$ acts on a lattice $\Gamma$, and we consider the possible actions of the corresponding affine semigroup $\mathcal{A} \times \Gamma$ on $\ell^{2}(\Gamma)$. We show in Section 2 that besides the natural action of translations and the "dilations with holes" (see e.g. [10, 19, 24]), several such actions may be constructed, based upon pseudodilations $\mathcal{D}_{a}, a \in \mathcal{A}$ acting on $\ell^{2}(\Gamma)$, generalizing the pseudodilations in [10] to the semigroup context. We introduce two families of pseudodilations (the principal pseudodilations and the associated pseudodilations), and investigate their properties. Such pseudodilations turn out to provide the appropriate setting for developing an abstract version of sub-band coding schemes.

We address in Section 3 the problem of existence of such pseudodilations in a more abstract setting. More precisely, we show that such a problem may be formulated as a problem 
of representation-valued semigroup cohomology. We develop the corresponding cohomology theory, and show that among the known multiresolution analyses, some may be obtained from trivial 1-cocycles, i.e. 1-coboundaries.

Finally, we address in Section 4 the problem of connection between the continuous and discrete approaches to wavelet analysis. We show that given a pseudodilation, the corresponding sampling of a continuous wavelet transform may be seen as a kind of generalized intertwiner between the action of the continuous affine group on $L^{2}(\mathbb{R})$ and an action of the discrete affine semigroup on $\ell^{2}(\mathbb{Z})$. ¿From our point of view, this approach may be understood as a first step towards a particular aspect of the harmonic analysis of the affine group, namely the decomposition of an irreducible representation of an affine group as a direct sum of representations of a discrete affine subsemigroup.

We notice that several different discrete versions of wavelet analysis have been considered. For the sake of simplicity, we limit the present discussion to the case of the multiresolution wavelet transforms as developed in I. Daubechies' book [9]. However, we emphasize that a similar approach may be developed in the case of the dyadic wavelet transform, as considered for example by S. Mallat [22], very much in the spirit of the Littlewood-Paley decompositions used by harmonic analysts since the early 30's. Notice also that the formalism developed in this paper does not seem, at the present stage,to yield directly new wavelet bases of sub-band coding schemes. This paper rather aims at analyzing the algebraic structures associated to multiresolution, so as to pave the way towards generalizations. The latter may for example include (separable or non separable) wavelet bases associated with nontrivial dilations in more than one dimension, or the wavelet bases associated to aperiodic tilings developed for studying quasicrystals in $[5,12]$, for which the semigroup to be considered is not a semidirect product any more.

\section{Pseudodilations and associated compatible filters}

\subsection{Background, notations}

Let $\mathcal{G}$ be an infinite locally compact abelian (LCA for short) group, $\Gamma \subset \mathcal{G}$ be a lattice in $\mathcal{G}$, and $\mathcal{A}$ be an abelian semigroup (see the Appendix for a survey of the main results in this area). We are interested in studying the possible actions of $\mathcal{A}$ on $\ell^{2}(\Gamma)$. We first need the following classical notions.

DeFinition 1 (Compatible action) 1. Let $\mathcal{A}$ be a semigroup and $\mathcal{E}$ a nonempty set. An action (or an operation) of $\mathcal{A}$ on $\mathcal{E}$ (on the left) is a mapping $(a, x) \in \mathcal{A} \times \mathcal{E} \rightarrow$ ax $\in \mathcal{E}$, such that:

(a) for all $a, a^{\prime} \in \mathcal{A}, x \in \mathcal{E}$

$$
a\left(a^{\prime} x\right)=\left(a a^{\prime}\right) x
$$

(b) whenever $\mathcal{A}$ contains a unit element 1 , we have for all $x \in \mathcal{E}$ :

$$
1 x=x .
$$

The action of $\mathcal{A}$ is one-to-one if for any $a \in \mathcal{A}$, the mapping $x \mapsto$ ax is one-to-one. 
2. Let $\mathcal{E}$ be a group and let $\mathcal{A}$ be a semigroup acting on $\mathcal{E}$. We say that the action of $\mathcal{A}$ on $\mathcal{E}$ is compatible (with the group law) if for any $a \in \mathcal{A}$,

$$
a(x y)=(a x)(a y), \quad \text { for all }(x, y) \in \mathcal{E} \times \mathcal{E} .
$$

DeFinition 2 (Divisibility) Let $\mathcal{A}$ be a semigroup operating on a lattice $\Gamma$ by a compatible and one-to-one action and let $a \in \mathcal{A}$. We say that a divides $\gamma \in \Gamma$ if there exists a (unique) $\gamma^{\prime} \in \Gamma$ such that $\gamma=a \gamma^{\prime}$. In such a case we write $a \mid \gamma$, and $\gamma^{\prime}=a^{-1} \gamma$.

REmark 1 Note that $a^{-1} \gamma$ only denotes an element $\gamma^{\prime}$ of $\Gamma$ such that $a \gamma^{\prime}=\gamma$; this doesno mean that $a$ is invertible in $\mathcal{A}$.

If $\mathcal{A}$ is an abelian semigroup with a one-to-one compatible action on a lattice $\Gamma$, then

$$
a a^{\prime}|\gamma \Longleftrightarrow a| \gamma \text { and } a^{\prime}\left|a^{-1} \gamma \Longleftrightarrow a^{\prime}\right| \gamma \text { and } a \mid a^{-1} \gamma
$$

and we have

$$
\left(a a^{\prime}\right)^{-1} \gamma=a^{-1}\left(a^{\prime-1} \gamma\right)=a^{\prime-1}\left(a^{-1} \gamma\right)
$$

Throughout Section 2, we shall always consider a generic situation consisting in a lattice $\Gamma$ in an infinite LCA group $\mathcal{G}$ and an abelian semigroup $\mathcal{A}$ with unit 1 acting on $\Gamma$, in such a way that the action of $\mathcal{A}$ on $\Gamma$ is compatible and one-to-one. In addition we suppose that for each $a \in \mathcal{A}, a \neq 1, a \Gamma$ is a lattice of $\mathcal{G}$ and the quotient $Q_{a}=\Gamma / a \Gamma$ is a nontrivial finite group, say

$$
Q_{a}=\left\{\gamma_{0}=0, \ldots, \gamma_{d(a)-1}\right\}
$$

where $d(a)=\left|Q_{a}\right|$ denotes the order of $Q_{a}(d(a)>1)$. It follows from the double coset theorem (see e.g. [21]) that

$$
d\left(a a^{\prime}\right) \geq d(a), \text { for all } a, a^{\prime} \in \mathcal{A}
$$

The action of $\mathcal{A}$ on $\Gamma$ induces an action on $\widehat{\Gamma}$, the dual action, in the natural way: for any $\gamma \in \Gamma, \chi \in \widehat{\Gamma}$, and $a \in \mathcal{A},\langle a \chi, \gamma\rangle=\langle\chi, a \gamma\rangle$. The dual action is compatible but non-necessary one-to-one.

By a slight abuse of language, we shall call sequences the functions on $\Gamma$.

ExAmple 1 Let $\mathcal{G}=\mathbb{R}$ be the real line. The (unitary) characters $\chi_{\theta}$ of $\mathbb{R}$ are given by the pure oscillations

$$
\left\langle\chi_{\theta}, t\right\rangle=e^{i \theta t}
$$

(see Appendix A for the notations and definitions). Each character of $\mathbb{R}$ can, therefore, be identified with a point $\theta \in \mathbb{R}$ which is interpreted as a frequency, and $\widehat{\mathbb{R}}$ is thus identified with $\mathbb{R}$. We take the Lebesgue measure $d x$ as the Haar measure on $\mathbb{R}$; if $d \theta$ denotes the Lebesgue measure on $\widehat{\mathbb{R}}$, then the dual measure of $d x$ is $\frac{d \theta}{2 \pi}$.

Let $\Gamma=\mathbb{Z}$; then $\Gamma^{\perp}=2 \pi \mathbb{Z}$. By Theorem A.1 we have $\widehat{\mathbb{Z}} \simeq \widehat{\mathbb{R}} / \mathbb{Z}^{\perp} ;$ the dual of $\mathbb{Z}$ can, therefore, be identified with the unit circle $\mathbb{T}=\mathbb{R} / 2 \pi \mathbb{Z}$. A fundamental domain of $\mathbb{Z}^{\perp}$ in $\widehat{\mathbb{R}}$ can be chosen to be $\Omega=[-\pi, \pi)$ and then $s\left(\mathbb{Z}^{\perp}\right)=1=s(\mathbb{Z})$. The Haar measure on $\mathbb{Z}$ is the counting measure and its dual measure on $\mathbb{T}$ is then $\frac{d \theta}{2 \pi}$. 
On the real line, the (continuous) dilation of parameter $a \in \mathbb{R}^{*}$ is the unitary operator $D_{a}$ on $L^{2}(\mathbb{R})$, defined by

$$
\left(D_{a} f\right)(x)=\frac{1}{\sqrt{|a|}} f\left(\frac{x}{a}\right)
$$

For $\Gamma=\mathbb{Z}$, there is no natural group of dilations acting on $\ell^{2}(\mathbb{Z})$. However, the multiplicative semigroup $\mathbb{Z}_{+}^{*}$, or any of its subsemigroups, acts naturally on $\mathbb{Z}$, and the action is compatible and one-to-one. We shall be interested in carrying such actions to $\ell^{2}(\mathbb{Z})$.

ExAmple 2 Let $\mathcal{G}=\mathbb{R}^{n}$, and consider the representation of $G L(n, \mathbb{R})$ on $L^{2}\left(\mathbb{R}^{n}\right)$ defined by

$$
\pi(h) f(x)=\frac{1}{\sqrt{|\operatorname{det} h|}} f\left(h^{-1} x\right)
$$

Take $\Gamma=\mathbb{Z}^{n}$. Let $\mathcal{A}$ be any abelian subsemigroup of $G L(n, \mathbb{R})$ consisting of matrices with integer entries (examples have been considered by $[4,11]$ in the group case). Suitable choices for $\mathcal{A}$ are, for instance, the semigroup of diagonal $n \times n$ invertible matrices with integer coefficients, or any abelian subsemigroup of $\mathbb{Z}_{+}^{*} \times O(n, Z)$, where $O(n, \mathbb{Z})$ is the group of orthogonal $n \times n$ matrices with integral coefficients, the so-called hyperoctahedral group [3]. $\mathcal{A}$ acts on $\Gamma$ in a natural way, and it is easily verified that the action is one-to-one and compatible. Another example is provided by $\mathcal{A}=\left\{A^{j}, j=0,1,2, \ldots\right\}$, where $A$ is any nonsingular matrix preserving $\mathbb{Z}^{n}$ whose (possibly complex) eigenvalues have modulus strictly greater than 1.

The need to construct discrete analogs of dilation operators has led the authors of [10] to introduce "pseudodilations" for sequences on $\mathbb{Z} / p \mathbb{Z}$, with $p$ a prime number. Such pseudodilations are all constructed from systems of so-called compatible filters. However, the construction is tied to the fact that the dilations on $\mathbb{Z} / p \mathbb{Z}$ form a group, which is not the case we consider here. Our goal is to construct on $\ell^{2}(\Gamma)$ an adapted version of the pseudodilation operators of $[10]$. We first set up the general framework.

\section{$2.2 \quad$ Principal pseudodilations}

A first candidate for dilations on $\ell^{2}(\Gamma)$ is provided in the following definition (we denote by $\mathcal{B}\left(\ell^{2}(\Gamma)\right)$ the set of bounded operators on $\left.\ell^{2}(\Gamma)\right)$.

Definition 3 (Natural dilation) Let $a \in \mathcal{A}$. The natural dilation, (or dilation with holes) on $\ell^{2}(\Gamma)$ is the mapping $\Delta: a \in \mathcal{A} \mapsto \Delta_{a} \in \mathcal{B}\left(\ell^{2}(\Gamma)\right)$, where $\Delta_{a}$ is defined as follows: for any sequence $\left\{u_{\gamma}, \gamma \in \Gamma\right\} \in \ell^{2}(\Gamma)$,

$$
\left(\Delta_{a} u\right)_{\gamma}=\left\{\begin{aligned}
u_{a^{-1} \gamma} & \text { if } a \mid \gamma \\
0 & \text { otherwise. }
\end{aligned}\right.
$$

For each $a \in \mathcal{A}$ we thus obtain a map $\Delta_{a}$ defined on the set of all sequence $u=\left(u_{\gamma}\right)_{\gamma \in \Gamma}$ which dilates them by inserting zeros. We shall denote again by $\Delta_{a}$ every restriction of this map. The maps $\Delta_{a}(a \in \mathcal{A})$ satisfy the following properties; the proof of each of them is obvious and is left to the reader.

- For all $a, a^{\prime} \in \mathcal{A}$,

$$
\Delta_{a} \Delta_{a^{\prime}}=\Delta_{a a^{\prime}} .
$$


- Let $T_{\gamma}$ be the translation of parameter $\gamma \in \Gamma$; we have

$$
T_{\gamma} \Delta_{a}=\Delta_{a} T_{a^{-1} \gamma} \quad \text { if } a \mid \gamma
$$

- If $u$ and $v$ are two sequences such that their convolution product exists then

$$
\Delta_{a}(u * v)=\left(\Delta_{a} u\right) *\left(\Delta_{a} v\right)
$$

- $\Delta_{a}$ is a non-surjective isometry on $\ell^{2}(\Gamma)$ and its adjoint is defined by:

$$
\left(\Delta_{a}^{*} u\right)_{\gamma}=u_{a \gamma}, \quad \text { for all } u \in \ell^{2}(\Gamma)
$$

The action of $\Delta_{a}$ in the Fourier domain is as follows: for all $u \in \ell^{2}(\Gamma)$,

$$
\widehat{\Delta_{a} u}(\chi)=\widehat{u}(a \chi), \quad \forall \chi \in \widehat{\Gamma} .
$$

The properties of $\Delta_{a}$ listed above are reminiscent of the main properties encountered when considering the affine group of the real line and the associated dilation. However, we shall see that the natural dilation is not the only candidate for a dilation on $\ell^{2}(\Gamma)$; in addition, the natural dilation is often not satisfactory for practical purposes, as will be clear from the next example.

ExAmple 1 (continued) Consider for instance the natural dilation by a factor $2, \Delta_{2}$, on $\ell^{2}(\mathbb{Z})$. The main difference between $\Delta_{2}$ and the continuously defined $D_{2}$ lies in the fact that, by construction, half of the coefficients of a sequence dilated using $\Delta_{2}$ vanish: $\left(\Delta_{2} f\right)_{2 k+1}=$ $0 \forall k \in \mathbb{Z}$. Therefore, a sequence dilated with $\Delta_{2}$ can hardly be interpreted as a sampling (perfect or imperfect) of a continuously defined function, dilated with $D_{2}$.

More generally, the fundamental difference between these $D_{a}$ and $\Delta_{a}$ is as follows: given a finite sequence $\left\{u_{\gamma}\right\}$, the measure of the support of a dilated sequence $\Delta_{a} u$, does not change, since the measure we use on $\Gamma$ is the counting measure. Therefore, it makes sense to look for alternative solutions. One of these is provided by the pseudodilations, defined as follows.

DeFINITION 4 (Pseudodilation) Let $\mathcal{A}$ be an abelian semigroup with unity, acting on a lattice $\Gamma \subset \mathcal{G}$, in such a way that the action is compatible and one-to-one. A pseudodilation (or principal pseudodilation) on $\ell^{2}(\Gamma)$ is a mapping

$$
\mathcal{D}: a \in \mathcal{A} \mapsto \mathcal{D}_{a}=K_{a} \Delta_{a} \in \mathcal{B}\left(\ell^{2}(\Gamma)\right)
$$

where $K_{a}$ is a bounded linear operator acting on $\ell^{2}(\Gamma)$, in such a way that

$$
\begin{aligned}
\mathcal{D}_{a} T_{\gamma} & =T_{a \gamma} \mathcal{D}_{a}, \quad a \in \mathcal{A}, \gamma \in \Gamma \\
\mathcal{D}_{a} \mathcal{D}_{a^{\prime}} & =\mathcal{D}_{a a^{\prime}}, \quad a, a^{\prime} \in \mathcal{A} .
\end{aligned}
$$

Notice that $\mathcal{D}_{1}=1$. To characterize the pseudodilations, we first need the following result. 
Lemma 1 Let $A$ be of the form $A=K \Delta_{a}$, with $a \in \mathcal{A}$ and $K$ a bounded linear operator on $\ell^{2}(\Gamma)$, such that

$$
A T_{\gamma}=T_{a \gamma} A, \quad \text { for all } \gamma \in \Gamma .
$$

Then there exists a sequence $\left\{k_{\gamma}, \gamma \in \Gamma\right\}$ such that for all $u \in \ell^{2}(\Gamma)$,

$$
(A u)_{\gamma}=\sum_{\gamma^{\prime} \in \Gamma} k_{\gamma-a \gamma^{\prime}} u_{\gamma^{\prime}} .
$$

Proof: Equation (2.16) reads

$$
\left[K, T_{a \gamma}\right] \Delta_{a}=0 .
$$

Writing, for any sequence $u=\left(u_{\gamma}\right)_{\gamma \in \Gamma} \in \ell^{2}(\Gamma), K u_{\gamma}=\sum k_{\gamma, \gamma^{\prime}} u_{\gamma^{\prime}}$ we see that (2.18) is equivalent to

$$
\sum k_{\gamma, a \gamma^{\prime}} u_{\gamma^{\prime}}=\sum k_{\gamma-a \gamma^{\prime}, 0} u_{\gamma^{\prime}}, \quad \gamma \in \Gamma
$$

which implies, for all $\gamma, \gamma^{\prime} \in \Gamma$

$$
k_{\gamma, a \gamma^{\prime}}=k_{\gamma-a \gamma^{\prime}, 0}:=k_{\gamma-a \gamma^{\prime}}
$$

for some sequence $\left\{k_{\gamma}, \gamma \in \Gamma\right\}$. Therefore, $K$ is the convolution by $k$.

Returning to the pseudodilation $\mathcal{D}$, we conclude that, for each $a \in \mathcal{A}$, there exists a sequence $\left\{h_{\gamma}^{(a)}, \gamma \in \Gamma\right\}$ such that, for all $u \in \ell^{2}(\Gamma)$,

$$
\left(\mathcal{D}_{a} u\right)_{\gamma}=\sum_{\gamma^{\prime} \in \Gamma} h_{\gamma-a \gamma^{\prime}}^{(a)} u_{\gamma^{\prime}}=\left(h^{(a)} *\left(\Delta_{a} u\right)\right)_{\gamma},
$$

and we shall write:

$$
\mathcal{D}_{a}=h^{(a)} * \Delta_{a}, \quad a \in \mathcal{A} .
$$

REMARK 2 If, instead of (2.13), we look for operators $\mathcal{D}_{a}$ of the form

$$
\mathcal{D}_{a}=\Delta_{a} M_{a}, \quad a \in \mathcal{A},
$$

then the relation (2.14) yields

$$
\Delta_{a}\left[M_{a}, T_{\gamma}\right]=0 \quad \forall a \in \mathcal{A}, \forall \gamma \in \Gamma,
$$

which implies that $M_{a}$ commutes with every translation of parameter $\gamma \in \Gamma$. A result of [6] then asserts that $M_{a}$ is a convolution operator on $\ell^{2}(\Gamma)$. Let $\mu^{(a)}$ be a sequence such that

$$
M_{a} v=\mu^{(a)} * v, \quad \text { for all } v \in \ell^{2}(\Gamma) .
$$

In this case,

$$
\mathcal{D}_{a} v=\left(\Delta_{a} \mu^{(a)}\right) *\left(\Delta_{a} v\right), \quad v \in \ell^{2}(\Gamma)
$$

and if we set $h^{(a)}=\Delta_{a} \mu^{(a)}$ and denote by $K_{a}$ the convolution by $h^{(a)}$, we find again (2.13) and (2.21). 
Let us consider the (group) Fourier transform of the sequences $h^{(a)}$ and set

$$
H_{a}=\widehat{h^{(a)}} .
$$

According to the discussion in Appendix A, $H_{a}$ is a function on the dual $\widehat{\Gamma}$ of $\Gamma$. Under Fourier transform the relations (2.21) read

$$
\widehat{\mathcal{D}_{a} u}(\chi)=H_{a}(\chi) \widehat{u}(a \chi) .
$$

Using (2.8) and (2.10), it is easy to check that (2.15) can be rewritten as follows: for all $a, a^{\prime} \in \mathcal{A}$

$$
h_{\gamma}^{\left(a a^{\prime}\right)}=\left(h^{(a)} * \Delta_{a} h^{\left(a^{\prime}\right)}\right)_{\gamma}=\sum_{\gamma^{\prime}} h_{\gamma-a \gamma^{\prime}}^{(a)} h_{\gamma^{\prime}}^{\left(a^{\prime}\right)},
$$

or equivalently in Fourier space: for all $\chi \in \widehat{\Gamma}$

$$
H_{a a^{\prime}}(\chi)=H_{a}(\chi) H_{a^{\prime}}(a \chi)
$$

We shall generically call filters operators of the form (2.21); the corresponding sequence $\left\{h_{\gamma}^{(a)}, \gamma \in \Gamma\right\}$ is the impulse response of the filter, and its Fourier transform $H_{a}$ is the transfer function of the filter. By abuse of language, we shall sometimes call the function $H_{a}$ a filter.

Example 3 We start here the discussion of an example that we shall revisit several times in what follows. Let $a \in \mathcal{A}$. We have in the dual space $\Gamma^{\perp} \subset(a \Gamma)^{\perp}$. As a result of the discussion at the end of Appendix A, and more precisely Eq. (A.101), we can choose fundamental domains $\Omega$ and $\Omega_{a}$ of $\Gamma^{\perp}$ and $(a \Gamma)^{\perp}$ in such a way that $\Omega_{a} \subset \Omega$ and

$$
\Omega=\bigcup_{\epsilon=0}^{d(a)-1}\left(\Omega_{a}+\chi_{\epsilon}\right),
$$

where the sets $\Omega_{a}+\chi_{\epsilon}$ intersect each other at most on sets of measure zero. In addition, $\Omega_{a}$ is a pre-image of $\Omega$ by $a$, in the sense that $\chi \in \Omega_{a}$ implies that $a \chi \in \Omega$.

Now, define the sequence $\left\{h_{\gamma}^{(a)}: \gamma \in \Gamma\right\}$ by its Fourier transform:

$$
H_{a}(\chi)=1_{\Omega_{a}}(\chi), \quad \chi \in \widehat{\mathcal{G}},
$$

where $1_{E}$ denotes the indicator function of the set $E$. Then the functions $H_{a}, a \in \mathcal{A}$ satisfy (2.25) by construction, and therefore the family $\left\{h^{(a)}: a \in \mathcal{A}\right\}$ satisfies (2.24). By analogy with the signal processing terminology, we shall call $\Omega_{a}$ a sub-band of $\Omega$.

\subsection{Associated pseudodilation}

Let $\mathcal{A}$ be an abelian semigroup with unit 1 , acting on a lattice $\Gamma \subset \mathcal{G}$, in such a way that the action is compatible and one-to-one, and for each $a \in \mathcal{A}$, let $Q_{a}$ be defined as in (2.4). As before, we assume that, for all $a \neq 1, Q_{a}$ is finite and nontrivial, so that $d(a)>1$. We now introduce the following 
DEFINITION 5 (Associated pseudodilation) Given a principal pseudodilation $\mathcal{D}$, an associated pseudodilation on $\ell^{2}(\Gamma)$ is a mapping $\widetilde{\mathcal{D}}$ which assigns to any pair $\left(a, a^{\prime}\right) \in \mathcal{A}^{2}, a^{\prime} \neq 1$, the family of linear operators $\widetilde{\mathcal{D}}_{a, a^{\prime} ; \epsilon}, \epsilon=1, \ldots, d\left(a^{\prime}\right)-1$, of the form

$$
\widetilde{\mathcal{D}}_{a, a^{\prime} ; \epsilon}=\widetilde{K}_{a, a^{\prime} ; \epsilon} \Delta_{a a^{\prime}}
$$

where $\tilde{K}_{a, a^{\prime} ; \epsilon}$ is a bounded linear operator acting on $\ell^{2}(\Gamma)$, such that

$$
\begin{aligned}
\widetilde{\mathcal{D}}_{a, a^{\prime} ; \epsilon} T_{\gamma} & =T_{\left(a a^{\prime}\right) \gamma} \widetilde{\mathcal{D}}_{a, a^{\prime} ; \epsilon}, \quad a, a^{\prime} \in \mathcal{A}, \gamma \in \Gamma \\
\mathcal{D}_{a_{0}} \widetilde{\mathcal{D}}_{a_{1}, a^{\prime} ; \epsilon} & =\widetilde{\mathcal{D}}_{a_{0} a_{1}, a^{\prime} ; \epsilon}, \quad a_{0}, a_{1}, a^{\prime} \in \mathcal{A} .
\end{aligned}
$$

REMARK 3 We emphasize the fact that an associated pseudodilation is not a pseudodilation in the sense of Definition 4. By a pseudodilation, we shall always mean a principal pseudodilation. Notice also that an associated pseudodilation $\widetilde{\mathcal{D}}$ is completely determined by the operators

$$
\widetilde{\mathcal{D}}_{a ; \epsilon}:=\widetilde{\mathcal{D}}_{1, a ; \epsilon}, \quad a \in \mathcal{A}, a \neq 1, \epsilon=1, \ldots d(a)-1
$$

and the relation $(2.30)$.

Using Lemma 1, we observe that for all $\left(a, a^{\prime}\right) \in \mathcal{A}^{2}, a^{\prime} \neq 1$ and $\epsilon=1, \ldots d\left(a^{\prime}\right)-1$, there exists a sequence $\left\{g_{\gamma}^{\left(a, a^{\prime} ; \epsilon\right)}, \gamma \in \Gamma\right\}$, such that the associated pseudodilation reads

$$
\left(\widetilde{\mathcal{D}}_{a, a^{\prime} ; \epsilon} u\right)_{\gamma}=\sum_{\gamma^{\prime} \in \Gamma} g_{\gamma-\left(a a^{\prime}\right) \gamma^{\prime}}^{\left(a, a^{\prime} ; \epsilon\right)} u_{\gamma^{\prime}}=\left(g^{\left(a, a^{\prime} ; \epsilon\right)} *\left(\Delta_{a a^{\prime}} u\right)\right)_{\gamma} .
$$

In addition, it follows directly from Definition 5 and Remark 3 that the sequences $\left\{g_{\gamma}^{\left(a, a^{\prime} ; \epsilon\right)}\right.$, $\gamma \in \Gamma\}$ must satisfy (and may actually be generated by) the following compatibility equations:

$$
g^{\left(a_{0} a_{1}, a^{\prime} ; \epsilon\right)}=h^{\left(a_{0}\right)} * \Delta_{a_{0}} g^{\left(a_{1}, a^{\prime} ; \epsilon\right)} .
$$

Let $G_{\left(a, a^{\prime} ; \epsilon\right)}$ denote the Fourier transform of the sequence $g^{\left(a, a^{\prime} ; \epsilon\right)}$. In the Fourier domain, the compatibility relations (2.32) read

$$
G_{a_{0} a_{1}, a^{\prime} ; \epsilon}(\chi)=H_{a_{0}}(\chi) G_{a_{1}, a^{\prime} ; \epsilon}\left(a_{0} \chi\right) .
$$

In particular, setting for simplicity $G_{a, \epsilon}=G_{1, a ; \epsilon}$, we have

$$
G_{a, a^{\prime} ; \epsilon}(\chi)=H_{a}(\chi) G_{a^{\prime} ; \epsilon}(a \chi)
$$

The arguments of this section and the previous one motivate the following definition:

Definition 6 (Compatible filters) A family $\left\{\left(H_{a}, G_{a, a^{\prime} ; \epsilon}\right), a, a^{\prime} \in \mathcal{A}, a^{\prime} \neq 1, \epsilon=1, \ldots, d\left(a^{\prime}\right)-1\right\}$ of bounded functions on $\widehat{\Gamma}$ satisfying the conditions (2.25) and (2.33) is called a family of compatible filters.

Therefore, we can summarize the results of this section as follows: 
THEOREM 1 Let $\mathcal{D}$ be a principal pseudodilation, and $\widetilde{\mathcal{D}}$ be an associated pseudodilation. Then they are necessarily filters of the form (2.21) and (2.31), and the corresponding transfer functions $\left\{\left(H_{a}, G_{a, a^{\prime} ; \epsilon}\right), a, a^{\prime} \in \mathcal{A}, \epsilon=1, \ldots, d\left(a^{\prime}\right)-1\right\}$ (functions on $\widehat{\Gamma}$ ) is a family of compatible filters.

REMARK 4 A sufficient condition for the pseudodilation $\mathcal{D}_{a}$ to be bounded is that the filters $H_{a} \in L^{\infty}(\widehat{\Gamma})$. In such cases we have $\left\|\mathcal{D}_{a} u\right\|_{\ell^{2}(\Gamma)} \leq\left\|H_{a}\right\|_{L^{\infty}(\widehat{\Gamma})}\|u\|_{\ell^{2}(\Gamma)}$. Similar bounds hold for $\widetilde{\mathcal{D}}$.

Since $h^{(a)}$ can be recovered from $H_{a}$, the complete description of the $\mathcal{D}_{a}$ is obtained if one can completely classify the solutions of (2.25).

EXAMPLE 4 (Natural pseudodilations) Let $a \in \mathcal{A}, a \neq 1$, and let us consider $Q_{a}=\Gamma / a \Gamma=$ $\left\{0, \ldots, \gamma_{d(a)-1}\right\}$. The impulse response of the natural dilation is clearly

$$
h_{\gamma}^{(a)}=\delta_{\gamma, 0}
$$

Let the sequences $g^{(a ; \epsilon)}, \epsilon=1, \ldots d(a)-1$, be defined according to

$$
g_{\gamma}^{(a ; \epsilon)}=\delta_{\gamma, \gamma_{\epsilon}},
$$

and define the other sequences $g^{\left(a, a^{\prime} ; \epsilon\right)}$ using Eq. (2.32). It is readily verified that the corresponding family of functions $G_{a, a^{\prime} ; \epsilon}$, together with the functions $H_{a}=1$ occuring in the case of natural dilations (see Definition 3 ), yield a family of compatible filters.

EXAMPLE 3 (continued) Let us come back to the example described above in Eq. (2.27). With Eq. (2.26) in mind, we define, for all $a, a^{\prime} \in \mathcal{A}, a^{\prime} \neq 1$ and $\epsilon=1, \ldots d\left(a^{\prime}\right)-1$, the functions

$$
G_{a, a^{\prime} ; \epsilon}(\chi)=1_{\Omega_{a a^{\prime}}}\left(\chi-\chi_{\epsilon}\right), \quad \chi \in \widehat{\Gamma} .
$$

Then it follows from the decomposition $\Omega_{a}=\bigcup_{\epsilon=0}^{d\left(a^{\prime}\right)-1}\left(\Omega_{a a^{\prime}}+\chi_{\epsilon}\right)$ that $G_{a, a^{\prime} ; \epsilon}(\chi)=G_{a^{\prime} ; \epsilon}(a \chi)$. Since both functions are supported in $\Omega_{a}$, and since $H_{a}(\chi)=1$ identically on $\Omega_{a}$, this in turn implies that the compatibility relation (2.34) is fulfilled. Therefore, the operators $\widetilde{\mathcal{D}}_{a, a^{\prime} ; \epsilon}$ defined by such filters $G_{a, a^{\prime} ; \epsilon}$ form a pseudodilation associated to $\mathcal{D}$.

\subsection{Adjoint pseudodilations and inversion}

Let us now consider the adjoint operators of the principal and associated pseudodilations defined in the previous sections. For $u \in \ell^{2}(\Gamma)$, we have

$$
\begin{aligned}
\left(\mathcal{D}_{a}^{*} u\right)_{\gamma} & =\sum_{\gamma^{\prime}} \bar{h}_{\gamma^{\prime}-a \gamma}^{(a)} u_{\gamma^{\prime}} \\
\left(\widetilde{\mathcal{D}}_{a, a^{\prime} ; \epsilon}^{*} u\right)_{\gamma} & =\sum_{\gamma^{\prime}} \bar{g}_{\gamma^{\prime}-\left(a a^{\prime}\right) \gamma}^{(a, ;)} u_{\gamma^{\prime}}
\end{aligned}
$$

Let us introduce at this point the synthesis operators, defined by

$$
\mathcal{S}_{a, a^{\prime}}=\mathcal{D}_{a a^{\prime}} \mathcal{D}_{a a^{\prime}}^{*}+\sum_{\epsilon=1}^{d\left(a^{\prime}\right)-1} \widetilde{\mathcal{D}}_{a, a^{\prime} ; \epsilon} \widetilde{\mathcal{D}}_{a, a^{\prime} ; \epsilon}^{*}
$$


and in particular

$$
\mathcal{S}_{a} \equiv \mathcal{S}_{1, a}=\mathcal{D}_{a} \mathcal{D}_{a}^{*}+\sum_{\epsilon=1}^{d(a)-1} \widetilde{\mathcal{D}}_{a ; \epsilon} \widetilde{\mathcal{D}}_{a ; \epsilon}^{*} .
$$

The following result is readily verified:

Lemma 2 The synthesis operators satisfy

$$
\mathcal{S}_{a, a^{\prime}}=\mathcal{D}_{a} \mathcal{S}_{a^{\prime}} \mathcal{D}_{a}^{*}, \quad \forall a, a^{\prime} \in \mathcal{A}
$$

Therefore, the synthesis operators are completely characterized by the operators $\mathcal{S}_{a}=\mathcal{S}_{1, a}, a \in$ $\mathcal{A}$.

Definition 7 (Sub-band coding scheme) A family of compatible filters define a sub-band coding scheme if for all $a \in \mathcal{A}, \mathcal{S}_{a}=1$.

Hence, given such a sub-band coding scheme, one may decompose any $s \in \ell^{2}(\Gamma)$ as follows. Let $s^{1}=s$, and define the sequences $s^{a}, a \in \mathcal{A}$ and $d^{a, a^{\prime} ; \epsilon}$ inductively by

$$
s^{a}=\mathcal{D}_{a}^{*} s, \quad d^{a, a^{\prime} ; \epsilon}=\widetilde{\mathcal{D}}_{a^{\prime} ; \epsilon}^{*} s^{a}
$$

Then, we have the "wavelet-like" decomposition:

$$
\begin{aligned}
s & =\mathcal{D}_{a} s^{a}+\sum_{\epsilon=1}^{d(a)-1} \widetilde{\mathcal{D}}_{a ; \epsilon} d^{a, 1 ; \epsilon} \\
& =\mathcal{D}_{a^{2}} s^{a^{2}}+\sum_{\epsilon=1}^{d(a)-1} \widetilde{\mathcal{D}}_{a, a ; \epsilon} d^{a, a ; \epsilon}+\sum_{\epsilon=1}^{d(a)-1} \widetilde{\mathcal{D}}_{a ; \epsilon} d^{1, a ; \epsilon}
\end{aligned}
$$

However, we can also write directly, for example,

$$
s=\mathcal{D}_{a^{2}} s^{a^{2}}+\sum_{\epsilon=1}^{d\left(a^{2}\right)-1} \widetilde{\mathcal{D}}_{1, a^{2} ; \epsilon} d^{1, a^{2} ; \epsilon}
$$

These two decompositions are of a different nature, for they make use of different operators $\widetilde{\mathcal{D}}_{u ; \epsilon}$. We shall see more precisely the difference between these two types of decompositions when discussing examples in the case $\ell^{2}(\mathbb{Z})$.

EXAMPle 3 (continued) Return to the example of the principal and associated pseudodilations defined by Eqs. (2.27) and (2.37). Then it is a direct consequence of Eq. (2.26) that, for all $a \in \mathcal{A}, a \neq 1$,

$$
\left|H_{a}(\chi)\right|^{2}+\sum_{\epsilon=1}^{d(a)-1}\left|G_{a ; \epsilon}(\chi)\right|^{2}=1 \quad \text { for all } \chi \in \widehat{\Gamma} .
$$

This implies that for all $a \in \mathcal{A}, \mathcal{S}_{a}=1$. Therefore, we have constructed a first example of sub-band coding scheme. 
Example 4 (continued) Coming back to the case of natural pseudodilations, we easily verify that with the impulse responses given in (2.35) and (2.36), we have

$$
\left(\mathcal{D}_{a} \mathcal{D}_{a}^{*} s\right)_{\gamma}= \begin{cases}s_{\gamma} & \text { if } a \mid \gamma \\ 0 & \text { otherwise }\end{cases}
$$

and

$$
\left(\widetilde{\mathcal{D}}_{a ; \epsilon} \widetilde{\mathcal{D}}_{a ; \epsilon}^{*} s\right)_{\gamma}= \begin{cases}s_{\gamma} & \text { if } a \mid\left(\gamma+\gamma_{\epsilon}\right) \\ 0 & \text { otherwise }\end{cases}
$$

In such a case, it is clear that $\mathcal{S}_{a}=1$, which yields again a sub-band coding scheme. We notice that this example is the abstract version of the scheme used in [17] to generate families of perfect reconstruction quadrature mirror filters.

REMARK 5 The definition of sub-band coding scheme we have given turns out to be too restrictive in many practical situations. A simple extension, which leads to biorthogonal multiresolution decompositions of sequences (and functions), consists in decoupling the decomposition and reconstruction steps, by using different families of compatible filters in those two steps. For example, let $(\mathcal{D}, \widetilde{\mathcal{D}})$ and $(\mathcal{C}, \widetilde{\mathcal{C}})$ be two pairs (pseudodilation, associated pseudodilation). Choosing, say, the pair $(\mathcal{D}, \widetilde{\mathcal{D}})$ for the decomposition and the pair $(\mathcal{C}, \widetilde{\mathcal{C}})$ for the reconstruction, the corresponding synthesis operators read

$$
\mathcal{S}_{a, a^{\prime}}=\mathcal{C}_{a, a^{\prime}} \mathcal{D}_{a, a^{\prime}}^{*}+\sum_{\epsilon=1}^{d\left(a^{\prime}\right)-1} \widetilde{\mathcal{C}}_{a, a^{\prime} ; \epsilon} \widetilde{\mathcal{D}}_{a, a^{\prime} ; \epsilon}^{*}
$$

and the pseudodilations form a biorthogonal sub-band coding scheme if for all $a \in \mathcal{A}$, we have $\mathcal{S}_{a}=1$. We shall not enter into the details of the biorthogonal sub-band coding schemes, which are (at least from the algebraic point of view) the direct generalizations of the classical ones. We simply notice that they offer extra freedom in the design of compatible perfect reconstruction filters.

\subsection{Compatible filters in the case $\mathcal{G}=\mathbb{R}, \mathcal{A} \subset \mathbb{Z}_{+}$}

Let us now specialize to the case of interest in classical one-dimensional wavelet theory, namely the case $\mathcal{G}=\mathbb{R}$ and $\Gamma=\mathbb{Z}$. Our goal here is twofold. We first want to make the connection with the classical multiresolution theory. We also take the opportunity to describe a few simple situations which will help understanding the next section.

Let $\mathcal{A}$ be any subsemigroup of the multiplicative semigroup $\mathbb{Z}^{*}$; let $\left\{\left(H_{a}, G_{a}\right), a \in \mathcal{A}\right\}$ be a system of compatible filters. After identifying the dual $\mathbb{T}$ of $\mathbb{Z}$ with the interval $[-\pi, \pi)$, the functions $H_{a}$ and $G_{a}$ on the dual $\mathbb{T}$ of $\mathbb{Z}$ are now considered as $2 \pi$-periodic functions.

We first notice that, given a $2 \pi$-periodic function $\Phi$, and setting, for all $a \in \mathcal{A}$,

$$
H_{a}(\theta)=\frac{\Phi(a \theta)}{\Phi(\theta)}
$$

we immediatly obtain a solution of (2.25), provided that the quotients are well-defined and define bounded $2 \pi$-periodic functions for all $a \in \mathcal{A}$. We call such families trivial, for reasons which will become clear in Section 3 below. We notice that Eq. (2.44) is reminiscent of the 
relationship between the Fourier transform of the scaling function and the low-pass filter in classical multiresolution theory. However, the identification is not completely adequate, for $\Phi(\theta)$ is $2 \pi$-periodic, unlike the Fourier transform of the scaling function.

More generally, any bounded function $\Phi \in L^{\infty}(\mathbb{R})$ such that the ratio (2.44) defines a $2 \pi$-periodic function for all $a \in \mathcal{A}$ also yields a solution of the compatibility relation (2.25). We shall come back to that point in Remark 7 below.

Let us also remark that besides the simple solutions of the form (2.44), we can also consider functions $H_{a}(\theta)$ of the form

$$
H_{a}(\theta)=a^{\alpha} \frac{\Phi(a \theta)}{\Phi(\theta)}
$$

(for some real number $\alpha$ ), which also satisfy the compatibility relations (2.25). This comes from the fact that $\left(a a^{\prime}\right)^{\alpha}=a^{\alpha} a^{\prime \alpha}$, in other words that $a \mapsto a^{\alpha}$ is a character of the semigroup $\mathcal{A}$.

It is obvious that the specific choice discussed in Example 3 is applicable to the present case without major difficulty. Identifying again the circle group $\widehat{\Gamma}=\mathbb{T}$ with the fundamental domain $\Omega=[-\pi, \pi)$, we set for all $a \in \mathcal{A}, \Omega_{a}=[-\pi / a, \pi / a) \subset \Omega$ and $d(a)=a$. Equation $(2.26)$ is trivially satisfied by setting

$$
\Omega_{a}+\chi_{\epsilon}=\left[-(\epsilon+1) \frac{\pi}{a},-\epsilon \frac{\pi}{a}\right] \cup\left[\epsilon \frac{\pi}{a},(\epsilon+1) \frac{\pi}{a}\right]
$$

Hence,

$$
H_{a}(\theta)=\sum_{n \in \mathbb{Z}} 1_{\left[-\frac{\pi}{a}, \frac{\pi}{a}\right]}(\theta-2 \pi n)
$$

and

$$
G_{a ; \epsilon}(\theta)=\sum_{n \in \mathbb{Z}} 1_{\Omega_{a}+\chi_{\epsilon}}(\theta-2 \pi n)
$$

generate families of compatible filters. In addition, for all $a \in \mathcal{A}$, we have that

$$
\left|H_{a}(\theta)\right|^{2}+\sum_{\epsilon=1}^{d(a)-1}\left|G_{a ; \epsilon}(\theta)\right|^{2}=1,
$$

which tells us that we are in the situation of a sub-band coding scheme. This particular case corresponds to the so-called Shannon multiresolution analyses of MRA theory, namely the multiresolution analysis associated to the classical sampling theorem (see [16] for a review).

Example 5 (Haar) Another simple example of such a "trivial" family of compatible filters is provided by the theory of spline functions. Let us again consider any subsemigroup $\mathcal{A} \subset \mathbb{Z}_{+}$, and define for all $a \in \mathcal{A}$

$$
h_{k}^{(a)}= \begin{cases}a^{-1} & \text { if } 0 \leq k \leq a-1 \\ 0 & \text { otherwise }\end{cases}
$$

A direct calculation yields

$$
H_{a}(\theta)=\frac{1}{a} \frac{1-e^{-i a \theta}}{1-e^{-i \theta}}
$$


It is easily verified that such a function satisfies Eq. (2.25). Notice that this is a solution of the form (2.45), with $\Phi(\theta)=1-e^{-i \theta}$. Now, for each $\epsilon=1, \ldots, a-1$, let

$$
G_{a ; \epsilon}(\theta)=H_{a}\left(\theta-\epsilon \frac{2 \pi}{a}\right)
$$

Using the trigonometric identity $\sum_{\epsilon=0}^{a-1} \sin (\theta+\epsilon \pi / a)^{-2}=a^{2} \sin (a \theta)^{-2}$ valid for all $a \in \mathbb{Z}_{+}$ and $\theta \in \mathbb{R}$, we easily see that Eq. (2.46) is satisfied. This tells us that we have constructed a sub-band coding scheme. In the classical wavelet literature, such a scheme goes under the name of the Haar MRA.

Example 6 (splines) The Haar MRA is the simplest instance of the so-called spline multiresolution analyses (see e.g. [8] or [13] for reviews; see also [25] for spline constructions in more general situations). The previous example suggests to study higher order spline filters. This may be done by defining the $H_{a}$ filters by

$$
H_{a}(\theta)=\frac{1}{a^{N}}\left(\frac{1-e^{-i a \theta}}{1-e^{-i \theta}}\right)^{N},
$$

and the $G_{a ; \epsilon}$ filters by Eq. (2.49). Since the filters $H_{a}$ again assume the form (2.45), the compatibility condition (2.25) is verified by construction. However, the perfect reconstruction condition (2.46) is not satisfied any more. Such a problem is usually addressed in two different ways: either by modifying the filter $H_{a}$ and $G_{a ; \epsilon}$ by multiplying them by a convenient periodic function, without destroying the compatibility relation (nor the form (2.45) in fact), or by going to a biorthogonal scheme, as alluded to in Remark 5 above.

By now, many more sub-band coding schemes have been proposed in the wavelet literature (see e.g [9, 27] for a non-exhaustive account; see also [18] for a generic method for generating such sub-band coding schemes). To our knowledge, most of them don't assume the simple forms (2.44) or (2.45). Understanding more precisely that point is the motivation of the next section.

\section{Cohomological interpretation}

The condition (2.25) satisfied by the transfer functions defining principal pseudodilations may be given an interesting cohomological interpretation, which we detail below. Our presentation follows the lines indicated in [2]. The reader who is only interested in the issues related to discrete and continuous wavelet and multiresolution analysis may skip the present section and proceed directly to Section 4 .

\subsection{Generalities}

Let us start by the following definition.

Definition $8 \mathcal{M}$ is a module over a semigroup $\mathcal{A}$ or an $\mathcal{A}$-module if $\mathcal{M}$ is an abelian group such that $\mathcal{A}$ acts on $\mathcal{M}$ by a compatible action. 
We shall be mostly interested in the following situation. Let $\Gamma$ be a lattice in a locally compact abelian group $\mathcal{G}$, and let $\mathcal{A}$ be an abelian semigroup acting on $\Gamma$. In this section we assume that the semigroup $\mathcal{A}$ operates on $\widehat{\Gamma}$ in such a way that the Haar measure $\mu_{\widehat{\Gamma}}$ on $\widehat{\Gamma}$ is quasiinvariant by $\mathcal{A}$. In other words, we assume that for all $a \in \mathcal{A}$, the mapping $E \mapsto \mu_{\widehat{\Gamma}}(a E)$ defines a positive measure on $\widehat{\Gamma}$, which is absolutely continuous with respect to $\mu_{\widehat{\Gamma}}$.

Let $\mathcal{R}$ be the commutative ring of all mappings of $\widehat{\Gamma}$ into $\mathbb{C}$

$$
\mathcal{R}=\operatorname{Map}(\widehat{\Gamma}, \mathbb{C})
$$

with the composition laws:

$$
\begin{aligned}
& \text { - Addition : }(\Phi, \Psi) \longmapsto \Phi+\Psi, \quad(\Phi+\Psi)(\chi)=\Phi(\chi)+\Psi(\chi) \\
& - \text { Multiplication }:(\Phi, \Psi) \longmapsto \Phi \Psi, \quad(\Phi \Psi)(\chi)=\Phi(\chi) \Psi(\chi)
\end{aligned}
$$

The subset $\mathcal{J}$ of $\mathcal{R}$ consisting of all mappings of $\Phi \in \mathcal{R}$ such that $\Phi(\chi)=0$ almost everywhere

on $\widehat{\Gamma}$ is an ideal in $\mathcal{R}$ and we consider the factor ring $\mathcal{R} / \mathcal{J}$. Now, let $\mathcal{M}$ be the group of units of $\mathcal{R} / \mathcal{J}$, that is the multiplicative group of all invertible elements of $\mathcal{R} / \mathcal{J}$. Clearly, $\mathcal{M}$ is the set of all the residue classes modulo $\mathcal{J}$ of the elements $\Phi \in \mathcal{R}$ such that $\Phi(\chi) \neq 0$ almost everywhere on $\widehat{\Gamma}$; we shall write again $\Phi \in \mathcal{M}$ without loss of generality.

We denote by 1 the unit element of $\mathcal{M}$ and by ${ }^{-1} \Phi$ the inverse of an element $\Phi \in \mathcal{M}$. We identify

$$
{ }^{-1}\left({ }^{-1} \Phi\right) \equiv{ }^{1} \Phi \equiv \Phi
$$

and we draw the reader's attention on the fact that in a product of the type $\Phi^{-1} \Psi$ with $\Phi, \Psi \in \mathcal{M}$, the " -1 " indicates the inverse of $\Psi$. If $\Phi_{1}, \ldots, \Phi_{n}$ are elements of $\mathcal{M}$, we denote their product by

$$
\prod_{j=1}^{n} \Phi_{j} \equiv \Phi_{1} \cdots \Phi_{n}
$$

The mapping

$$
(a, \Phi) \longmapsto a \Phi, \quad a \in \mathcal{A}, \Phi \in \mathcal{M} \quad \text { with } \quad(a \Phi)(\chi)=\Phi(a \chi) \quad \text { for all } \quad \chi \in \widehat{\Gamma}
$$

defines a compatible action of $\mathcal{A}$ on $\mathcal{M}$. $\mathcal{M}$ is therefore a module over the semigroup $\mathcal{A}$.

\subsection{The module of $n$-cochains}

For each integer $n \geq 1$, let $H^{n}$ be a function which maps ordered $n$-tuples of elements of $\mathcal{A}$ into $\mathcal{M}$, that is

$$
\begin{aligned}
H^{n}: \underbrace{\mathcal{A} \times \cdots \times \mathcal{A}}_{n} \longrightarrow \mathcal{M} & \\
\left(a_{1}, \ldots, a_{n}\right) \longmapsto H^{n}\left(a_{1}, \ldots, a_{n}\right) & :=H^{n}\left(a_{1}, \ldots, a_{n}\right) ? ? ? ? ? ? ? \\
& \equiv H_{\left(a_{1}, \ldots, a_{n}\right)}^{n}
\end{aligned}
$$

Such a function $H^{n}$ is called a $n$-cochain. We denote by $\mathcal{C}^{n}(\mathcal{A}, \mathcal{M}) \equiv \mathrm{C}^{n}$ the set of all $n$ cochains; for $n=0$, we define

$$
\varrho^{0}(\mathcal{A}, \mathcal{M}):=\mathcal{M}
$$


i.e., the 0 -cochains are elements the of $\mathcal{M}$.

The $\mathcal{A}$-module structure of $\mathcal{M}$ is transferred to $\mathfrak{C}^{n}$ in a natural way; indeed, $\mathfrak{C}^{n}$ is an abelian group under the composition law

$$
\left(U^{n}, V^{n}\right) \longmapsto U^{n} V^{n}
$$

where

$$
\left(U^{n} V^{n}\right)\left(a_{1}, \ldots, a_{n}\right):=U_{\left(a_{1}, \ldots, a_{n}\right)}^{n} V_{\left(a_{1}, \ldots, a_{n}\right)}^{n}
$$

As a matter of notation, we write again 1 for the unit element of $\mathrm{C}^{n}$ and ${ }^{-1} H^{n}$ for the inverse of a given $n$-cochain $H^{n}$. Hence, ${ }^{-1} H^{n}$ is defined by:

$$
{ }^{-1} H^{n}\left(a_{1}, \ldots, a_{n}\right)={ }^{-1} H_{\left(a_{1}, \ldots, a_{n}\right)}^{n}
$$

As we have done for the elements of $\mathcal{M}$, we identify

$$
{ }^{-1}\left({ }^{-1} H^{n}\right) \equiv{ }^{1} H^{n} \equiv H^{n}
$$

The semigroup $\mathcal{A}$ acts on $\mathfrak{C}^{n}$ by

$$
\left[a\left(H^{n}\right)\right]\left(a_{1}, \ldots, a_{n}\right):=a\left(H_{\left(a_{1}, \ldots, a_{n}\right)}^{n}\right)
$$

and one can easily check that this action is compatible with the group law of $\mathrm{e}^{n}$; thus, $\mathrm{C}^{n}(\mathcal{A}, \mathcal{M})$ is an $\mathcal{A}$-module for all $n \geq 0$.

\subsection{Differentials and cohomology groups}

Let $n \geq 0$ be an integer and $i \in\{0, \ldots, n+1\}$. Consider the map $p_{n+1}^{i}: \mathfrak{C}^{n}(\mathcal{A}, \mathcal{M}) \longrightarrow$ $\mathrm{e}^{n+1}(\mathcal{A}, \mathcal{M})$ given by $p_{n+1}^{i}\left(H^{n}\right)=H^{n+1}$, where $H^{n+1}$ is defined by

$$
H^{n+1}\left(a_{1}, \ldots, a_{n+1}\right)= \begin{cases}\left(a_{1} H^{n}\right)\left(a_{2}, \ldots, a_{n+1}\right) & \text { if } i=0 \\ H^{n}\left(a_{1}, \ldots, a_{i} a_{i+1}, \ldots, a_{n+1}\right) & \text { if } 0<i<n+1 \\ H^{n}\left(a_{1}, \ldots, a_{n}\right) & \text { if } i=n+1\end{cases}
$$

Proposition $1 p_{n+1}^{i}$ is a group homomorphism, for each integer $n \geq 0$ and each $i \in\{0, \ldots, n+$ $1\}$

Proof: This follows immediately from the definition of the composition law of $\mathrm{e}^{n}(\mathcal{A}, \mathcal{M})$ and the compatibility of the action of $\mathcal{A}$ on $\mathrm{e}^{n}(\mathcal{A}, \mathcal{M})$

We now use the maps $p_{n+1}^{i}$ to define the so-called differentials $\partial_{n}: \mathfrak{C}^{n}(\mathcal{A}, \mathcal{M}) \longrightarrow \mathfrak{C}^{n+1}(\mathcal{A}, \mathcal{M})$ $(n \geq 0)$ by

$$
\partial_{n}\left(H^{n}\right):=\prod_{i=0}^{n+1}{ }^{\varepsilon(n+i)}\left[p_{n+1}^{i}\left(H^{n}\right)\right]
$$

where $\varepsilon(k)=(-1)^{k}$, with $k$ an integer. We have the following fundamental result: 
Proposition $2 \partial_{n}$ is a group homomorphism for each integer $n \geq 0$, and

$$
\operatorname{Im}\left(\partial_{n} \circ \partial_{n-1}\right)=\{\mathbf{1}\}
$$

where Im denotes the image.

Proof: The fact that $\partial_{n}$ is a homomorphism follows from a straightforward verification which we leave to the reader. To prove that

$$
\operatorname{Im}\left(\partial_{n} \circ \partial_{n-1}\right)=\{\mathbf{1}\},
$$

we write, for the sake of simplicity,

$$
\partial_{n}=\prod_{i=0}^{n+1}{ }^{\varepsilon(n+i)}\left[p_{n+1}^{i}\right], \quad \text { for all } \quad n \geq 0
$$

hence, for $n \geq 1$,

$$
\begin{aligned}
\partial_{n} \circ \partial_{n-1} & =\left(\prod_{i=0}^{n+1} \varepsilon(n+i)\left[p_{n+1}^{i}\right]\right) \circ\left(\prod_{j=0}^{n}{ }^{\varepsilon(n+j-1)}\left[p_{n}^{j}\right]\right) \\
& =\prod_{i=1}^{n+1} \prod_{j=1}^{\varepsilon} \varepsilon(i+j-1)\left[p_{n+1}^{i} \circ p_{n}^{j}\right] \\
& =\prod_{0 \leq i \leq j \leq n}^{\varepsilon(i+j-1)}\left[p_{n+1}^{i} \circ p_{n}^{j}\right] \prod_{0 \leq j<i \leq n+1}{ }^{\varepsilon(i+j-1)}\left[p_{n+1}^{i} \circ p_{n}^{j}\right] .
\end{aligned}
$$

After rearranging terms, we obtain the following product

$$
\partial_{n} \circ \partial_{n-1}=\prod_{0 \leq i \leq j \leq n}^{\varepsilon(i+j)}\left[-1\left(p_{n+1}^{i} \circ p_{n}^{j}\right)\left(p_{n+1}^{j+1} \circ p_{n}^{i}\right)\right],
$$

which splits into seven types of factors, namely: (1) $i=j=0$; (2) $i=0$ and $j=n$; (3) $i=j=n$; (4) $0<i=j<n$; (5) $i=0$ and $0<j<n$; (6) $0<i<j<n$; and (7) $0<i<j=n$. For each factor, an explicit computation yields

$$
\left.{ }^{-1}\left(p_{n+1}^{i} \circ p_{n}^{j}\right)\left(p_{n+1}^{j+1} \circ p_{n}^{i}\right)\right]\left(H^{n-1}\right)=\mathbf{1}, \quad \text { for all } \quad H^{n-1} \in \mathcal{C}^{n-1}(\mathcal{A}, \mathcal{M}) .
$$

We only show as an example the computation of one of these factors, namely the type 6 ; the others are obtained in the same way. For $0<i<j<n$, the corresponding factors are

$$
{ }^{\varepsilon(i+j)}\left[-1\left(p_{n+1}^{i} \circ p_{n}^{j}\right)\left(p_{n+1}^{j+1} \circ p_{n}^{i}\right)\right] \quad(0<i<j<n) .
$$

On the one hand, we have

$$
\begin{aligned}
\left(p_{n+1}^{j+1} \circ p_{n}^{i}\right)\left(H^{n-1}\right)\left(a_{1}, \ldots, a_{n+1}\right) & =\left(p_{n}^{i} H^{n-1}\right)\left(a_{1}, \ldots, a_{j} a_{j+1}, \ldots, a_{n}\right) \\
& =\left(H^{n-1}\right)\left(a_{1}, \ldots, a_{i} a_{i+1}, \ldots, a_{j} a_{j+1} \ldots, a_{n}\right),
\end{aligned}
$$


and on the other hand

$$
\begin{aligned}
\left(p_{n+1}^{i} \circ p_{n}^{j}\right)\left(H^{n-1}\right)\left(a_{1}, \ldots, a_{n+1}\right) & =p_{n}^{j}\left(H^{n-1}\right)\left(a_{1}, \ldots, a_{i} a_{i+1}, \ldots, a_{n+1}\right) \\
& =\left(H^{n-1}\right)\left(a_{1}, \ldots, a_{i} a_{i+1}, \ldots, a_{j} a_{j+1}, \ldots, a_{n}\right)
\end{aligned}
$$

and the result follows; this concludes the proof.

We set

$$
\begin{aligned}
& \mathcal{Z}^{n}(\mathcal{A}, \mathcal{M}):=\operatorname{Ker}\left(\partial_{n}\right), \\
& \mathcal{B}^{n}(\mathcal{A}, \mathcal{M}):=\operatorname{Im}\left(\partial_{n-1}\right) \text {. }
\end{aligned}
$$

Following the usual terminology, the elements of $\mathcal{Z}^{n}(\mathcal{A}, \mathcal{M})$ are called $n$-cocycles and those of $\mathcal{B}^{n}(\mathcal{A}, \mathcal{M}), n$-coboundaries. For each $n \geq 1$, the quotient group

$$
\mathcal{H}^{n}(\mathcal{A}, \mathcal{M}):=\mathcal{Z}^{n}(\mathcal{A}, \mathcal{M}) / \mathcal{B}^{n}(\mathcal{A}, \mathcal{M})
$$

is the $n$th cohomology group of $\mathcal{A}$ in $\mathcal{M}$, and it measures the deviation from exactness of the complex

$$
\mathrm{C}^{0} \stackrel{\partial_{0}}{\longrightarrow} \mathrm{C}^{1} \stackrel{\partial_{1}}{\longrightarrow} \mathrm{C}^{2} \longrightarrow \ldots \longrightarrow \mathrm{C}^{n-1} \stackrel{\partial_{n-1}}{\longrightarrow} \mathrm{C}^{n} \stackrel{\partial_{n}}{\longrightarrow} \mathrm{C}^{n+1} \longrightarrow \ldots
$$

For characteriing the compatible filters, we only need the first cohomology group.

\subsection{Cohomological solution of the pseudodilation filters equations}

Let us start by first exhibiting the explicit form of $\partial_{0}$ and $\partial_{1}$ by means of (3.54). For $n=0$, the differential $\partial_{0}: \mathcal{M} \longrightarrow \mathcal{C}^{1}(\mathcal{A}, \mathcal{M})$ is defined as follows. For all $\Phi \in \mathcal{M}$ and $a \in \mathcal{A}$,

$$
\begin{aligned}
{\left[\partial_{0}(\Phi)\right](a) } & ={ }^{-1}\left[p_{1}^{1}(\Phi)(a)\right]\left[p_{1}^{0}(\Phi)(a)\right] \\
& \left.={ }^{-1} \Phi\right][a(\Phi)]=\left({ }^{-1} \Phi\right)(a \Phi),
\end{aligned}
$$

that is, for all $\Phi \in \mathcal{M}$ and $a \in \mathcal{A}$,

$$
\left[\partial_{0}(\Phi)\right](a)(\chi)=\frac{\Phi(a \chi)}{\Phi(\chi)}, \text { for all } \chi \in \widehat{\Gamma}
$$

For $n=1$, the differential $\partial_{1}: \mathcal{C}^{1} \longrightarrow \mathcal{C}^{2}$ reads: for all $H \in \mathcal{C}^{1}(\mathcal{A}, \mathcal{M})$ and $\left(a, a^{\prime}\right) \in \mathcal{A} \times \mathcal{A}$,

$$
\left[\partial_{1}(H)\right]\left(a, a^{\prime}\right)(\chi)=\frac{H_{a a^{\prime}}(\chi)}{H_{a}(\chi) H_{a^{\prime}}(a \chi)}, \quad \chi \in \widehat{\Gamma} .
$$

It is clear that each family $\left\{H_{a}: a \in \mathcal{A}\right\}$ of elements of $\mathcal{M}$ is uniquely identified with an element $H \in \mathrm{C}^{1}(\mathcal{A}, \mathcal{M})$ and vice versa.

We say that an element $H \in \mathcal{C}^{1}$ belongs to $\mathcal{L}^{\infty}(\mathcal{A}, \widehat{\Gamma})$ if and only if $H_{a}$ belongs to $L^{\infty}(\widehat{\Gamma})$ for all $a \in \mathcal{A}$. It then follows from Eqs. (2.25) and (3.56) that an element $H \in \mathrm{e}^{1}(\mathcal{A}, \mathcal{M})$ generates a pseudodilation if and only if $H \in \mathcal{L}^{\infty}(\mathcal{A}, \widehat{\Gamma})$ and

$$
\left[\partial_{1}(H)\right]\left(a, a^{\prime}\right)(\chi)=1, \quad \text { for all }\left(a, a^{\prime}\right) \in \mathcal{A}^{2} \quad \text { and } \chi \in \widehat{\Gamma},
$$


i.e.,

$$
\left\{\begin{array}{l}
{\left[\partial_{1}(H)\right]\left(a, a^{\prime}\right)=\mathbf{1}, \quad \forall\left(a, a^{\prime}\right) \in \mathcal{A}^{2}} \\
H \in \mathcal{L}^{\infty}(\mathcal{A}, \widehat{\Gamma}) .
\end{array}\right.
$$

Thus, we have proved that

Proposition 3 The set of all families of compatible filters indexed by $\mathcal{A}$ is $\operatorname{Ker}\left(\partial_{1}\right) \cap \mathcal{L}^{\infty}(\mathcal{A}, \widehat{\Gamma})$, where Ker denotes the kernel.

REmark 6 Let $H$ be an element of $\mathcal{C}^{1}$ satisfying (3.57); it is easy to verify that $(a H)$ also satisfies (3.57), for all $a \in \mathcal{A}$. Thus, $\operatorname{Ker}\left(\partial_{1}\right) \cap \mathcal{L}^{\infty}(\mathcal{A}, \widehat{\Gamma})$ is globally invariant under the action of the semigroup $\mathcal{A}$ on $\mathrm{C}^{1}$.

Let $H$ be an element of $\mathcal{C}^{1}$ which defines a family of compatible filters; it is interesting to know how to define explicitly the functions $H_{a}$ for a given $a \in \mathcal{A}$. In fact the answer is known for a particular class of solutions and is contained in Proposition 2. Indeed, by Proposition 2 , we have $\mathcal{B}^{1}(\mathcal{A}, \mathcal{M}) \subset \operatorname{Ker}\left(\partial_{1}\right)$; the relation (3.55) then shows that the family of functions $\left\{H_{a}, a \in \mathcal{A}\right\}$ defined by

$$
H_{a}(\chi)=\frac{\Phi(a \chi)}{\Phi(\chi)}
$$

is a system of compatible filters, where $\Phi$ is some element of $\mathcal{M}$ such that $H_{a}$ belongs to $L^{\infty}(\widehat{\Gamma})$. Notice that the compatible filters defined in Example 3 trivially belong to this class of solutions: in that case we even have that $H_{a}(\chi)=\Phi(a \chi)$. More generally, the classification of compatible filters would require to compute $\mathcal{H}^{1}(\mathcal{A}, \mathcal{M})$. This problem, which is still an open problem in wavelet analysis even in the simple case $\mathcal{G}=\mathbb{R}, \mathcal{A}=\left\{2^{j}, j=0,1 \ldots\right\}$, is also interesting by itself from the point of view of cohomology theory. Conversely, one may also hope that the tools of cohomology theory will help to classify the wavelet filters.

solutions

REMARK 7 Instead of considering the ring $\mathcal{R}$ as defined in (3.51), we could also have considered the ring $\mathcal{R}^{\prime}=\operatorname{Map}(\widehat{\mathcal{G}}, \mathbb{C})$ of mappings from $\widehat{\mathcal{G}}$ to $\mathcal{C}$ and develop the corresponding cohomology theory. In such a case, the differentials assume the same form. In particular,

$$
\left[\partial_{0}(\phi)\right](a)(\chi)=\frac{\phi(a \chi)}{\phi(\chi)}, \chi \in \widehat{\mathcal{G}},
$$

and

$$
\left[\partial_{1} H\left(a, a^{\prime}\right)\right](\chi)=\frac{H_{a a^{\prime}}(\chi)}{H_{a}(\chi) H_{a^{\prime}}(a \chi)}, \quad \chi \in \widehat{\mathcal{G}} .
$$

The connection with compatible filters in this case may be obtained by restricting to the 1cochains which may be defined on $\widehat{\Gamma}$. The corresponding cohomology problem is different from the preceding one. Notice that Eq. (3.59) is very suggestive from the wavelet point of view, since it is exactly the two-scale equation satisfied by the scaling function, expressed in the Fourier domain. However, let us stress again that an arbitrary coboundary of the cohomology constructed on $\widehat{\mathcal{G}}$ need not be well defined on $\widehat{\Gamma}$, thus is not a cochain for the cohomology constructed on $\widehat{\Gamma}$. The precise relationship between these two cohomology theories is another interesting open problem. 


\section{Discretized wavelet transform and generalized pyra- mid algorithms}

In the present section we show how the pseudodilation operators and compatible filters defined in Definition 4 introduce themselves naturally in the context of discretization of the continuous wavelet transform. For the sake of simplicity, we limit the present discussion to the one-dimensional situation. We shall see that discretization appears as a sort of generalized intertwining operator between the canonical action of the affine group on $L^{2}(\mathbb{R})$ and the action of some affine-type semigroups on $\ell^{2}(\mathbb{Z})$. As a by-product, this approach also provides a geometrical interpretation of the pyramid algorithms which have been developed for the computation of the wavelet coefficients, and a generalization for arbitrary integral scale.

Before going to more wavelet-related issues, let us start by specifying the action of the pseudodilations on continuously defined functions in the abstract setting.

\subsection{Action of the filters on functions}

Up to now, we have only worked at the level of sequences defined on a lattice $\Gamma$. To proceed further, our first step will be to transport the action of the filters on $L^{2}(\mathcal{G})$. In order to do that, we need additional assumptions. From now on, we will assume that the semigroup $\mathcal{A}$ under consideration also acts on $\mathcal{G}$, and that the action is compatible and one-to-one. Furthermore, we assume also that the action of $\mathcal{A}$ on $\mathcal{G}$ is such that for all $g \in \mathcal{G}$ and $a \in \mathcal{A}$, there is some $g^{\prime} \in \mathcal{G}$ such that $g=a g^{\prime}$. Finally, we assume that the Haar measure $\mu=\mu_{\mathcal{G}}$ on $\mathcal{G}$ is $\mathcal{A}$-quasi-invariant, i.e., that, for all $a \in \mathcal{A}$, the map $E \mapsto \mu(a E)$ defines a measure on $\mathcal{G}$, absolutely continuous with respect to $\mu$. Let us denote by

$$
\rho_{a}(g)=\frac{d \mu(a g)}{d \mu(g)}
$$

the corresponding Radon-Nikodym derivative which satisfies the cocycle condition: for all $a, a^{\prime} \in \mathcal{A}$ and $g \in \mathcal{G}$,

$$
\rho_{a a^{\prime}}(g)=\rho_{a}(g) \rho_{a^{\prime}}(a g) .
$$

Notice that the translation invariance of the Haar measure $\mu$ implies that for all $\gamma \in \Gamma$,

$$
\frac{d \mu(a g-\gamma)}{d \mu(g)}=\rho_{a}(g)
$$

Then we also have $\rho_{a^{\prime}}(a g-\gamma)=\rho_{a^{\prime}}(a g)$ for all $a, a^{\prime} \in \mathcal{A}, g \in \mathcal{G}, \gamma \in \Gamma$.

REMARK 8 Note the similarity of Eq. (4.62) with the compatibility condition (2.25) we already encountered. Both are of a similar nature, namely they describe deformations of the action of $\mathcal{G}$ (or $\Gamma$ ) by $\mathcal{A}$. However, the cocycles are defined on different spaces.

Let now $\mathcal{D}$ and $\widetilde{\mathcal{D}}$ be a principal and associated pseudodilations, and let $H_{a}$ and $G_{a, a^{\prime} ; \epsilon}$ be the corresponding filters, assumed to be bounded. Let us introduce the following operators, acting on $L^{2}(\mathcal{G})$ :

$$
\begin{aligned}
\mathcal{U}_{a} f(g) & =\rho_{a}(g) \sum_{\gamma} h_{\gamma}^{(a)} f(a g-\gamma) \\
\mathcal{V}_{a, a^{\prime} ; \epsilon} f(g) & =\rho_{a a^{\prime}}(g) \sum_{\gamma} g_{\gamma}^{\left(a, a^{\prime} ; \epsilon\right)} f\left(a a^{\prime} g-\gamma\right)
\end{aligned}
$$


These operators are the $L^{2}(\mathcal{G})$ analogues of the discrete operators $\mathcal{D}_{a}$ and $\widetilde{\mathcal{D}}_{a ; \epsilon}$, and enable us to transport the action of the pseudodilations onto $L^{2}(\mathcal{G})$. The following is a direct consequence of the compatibility relations and the cocycle condition (4.62):

COROLlaRY 1 The operators $\mathcal{U}_{a}$ and $\mathcal{V}_{a ; \epsilon}$ satisfy

$$
\begin{aligned}
\mathcal{U}_{a a^{\prime}} & =\mathcal{U}_{a} \mathcal{U}_{a^{\prime}} \\
\mathcal{V}_{a, a^{\prime} ; \epsilon} & =\mathcal{U}_{a} \mathcal{V}_{1, a^{\prime} ; \epsilon}
\end{aligned}
$$

In the Fourier domain, the operators $\mathcal{U}_{a}$ and $\mathcal{V}_{a ; \epsilon}$ read

$$
\begin{aligned}
\widehat{\mathcal{U}_{a} f}(a \chi) & =H_{a}(\chi) \widehat{f}(\chi) \\
\widehat{\mathcal{V}_{a ; \epsilon} f}(a \chi) & =G_{a ; \epsilon}(\chi) \widehat{f}(\chi)
\end{aligned}
$$

Finally, the counterpart of the perfect reconstruction property is given by the following

Corollary 2 Assume that the filters $H_{a}$ and $G_{a ; \epsilon}$ generate a sub-band coding scheme, in the sense of Definition 7 . Then we have the equality

$$
\mathcal{U}_{a}^{*} \mathcal{U}_{a}+\sum_{\epsilon=1}^{d(a)-1} \mathcal{V}_{a ; \epsilon}^{*} \mathcal{V}_{a ; \epsilon}=1
$$

These properties motivate the following definition.

DeFinition 9 Let $\left\{H_{a}, a \in \mathcal{A}\right\}$ be a family of filters satisfying the compatibility condition (2.25). A scaling function associated to the family $\left\{H_{a}, a \in \mathcal{A}\right\}$ is a square integrable function $\phi \in L^{2}(\mathcal{G})$ which is a fixed point of the operators $\mathcal{U}_{a}$ for all $a \in \mathcal{A}$.

Given a scaling function $\phi(g)$ and an associated pseudodilation $\widetilde{\mathcal{D}}$, one can then construct the associated generalized wavelets, defined by

$$
\psi^{a ; \epsilon}(g)=\mathcal{V}_{a ; \epsilon} \psi(g),
$$

and develop an abstract theory of wavelets. We refrain from doing so in this paper, and rather come back to the usual situation.

\subsection{The case $\mathcal{G}=\mathbb{R}, \Gamma=\mathbb{Z}$ and $\mathcal{A} \subset \mathbb{Z}_{+}$}

Let us come back to the case $\mathcal{G}=\mathbb{R}, \Gamma=\mathbb{Z}$. We denote by $\langle\cdot \mid \cdot\rangle(\operatorname{resp} .(\cdot \mid \cdot))$ the inner product of $L^{2}(\mathcal{G})\left(\operatorname{resp} . \ell^{2}(\Gamma)\right)$.

Let $\mathcal{A} \subset \mathbb{Z}_{+}^{*}$ be a multiplicative semigroup. The Lebesgue measure (which is also the Haar measure) on $\mathbb{R}$ is $\mathcal{A}$-quasi-invariant, and the corresponding Radon-Nikodym derivative is

$$
\rho_{a}(t)=a, \quad a \in \mathcal{A}, t \in \mathbb{R} .
$$

Let $\left\{\left(H_{a}, G_{a, a^{\prime} ; \epsilon}\right): a, a^{\prime} \in \mathcal{A}, \epsilon=1, \ldots d\left(a^{\prime}\right)-1\right\}$ be a system of compatible filters labelled by $\mathcal{A}$. Let us assume that there exists a scaling function $\phi \in L^{1}(\mathbb{R}) \cap L^{2}(\mathbb{R})$, with $\int \phi(t) d t=1$, 
satisfying the following two requirements:

- Stability: the functions $\phi(t-k), k \in \mathbb{Z}$ form a frame of their closed linear span $V$. In other words, there exist two constants $0<A \leq B<\infty$ such that for all $f \in V$, we have

$$
A\|f\|^{2} \leq \sum_{k}|\langle f, \phi(\cdot-k)\rangle|^{2} \leq B\|f\|^{2}
$$

- Refinability: ${ }^{1}$ for all $a \in \mathcal{A}$,

$$
\phi(t)=a \sum_{\ell} h_{\ell}^{(a)} \phi(a t-\ell)
$$

Then it follows from (4.71) that for all $\omega$,

$$
\widehat{\phi}(a \theta)=H_{a}(\theta) \widehat{\phi}(\theta), \quad \forall a \in \mathcal{A}, \theta \in \mathbb{R} .
$$

REMARK 9 Notice that formally, Equation (4.72) and the normalization condition define the function $\phi$ : for all $a \in \mathcal{A}, a \neq 1$ we may write

$$
\widehat{\phi}(\theta)=H_{a}\left(\frac{\theta}{a}\right) H_{a}\left(\frac{\theta}{a^{2}}\right) H_{a}\left(\frac{\theta}{a^{3}}\right) \ldots
$$

However, the convergence of such infinite products to "nice" $L^{2}$ functions is often problematic. We shall not discuss such issues here.

Let us now consider an associated pseudodilation $\widetilde{\mathcal{D}}$, and denote as before by $\left\{g^{\left(a, a^{\prime} ; \epsilon\right)}\right\}$ the impulse responses of the corresponding filters. According to (4.69), we introduce the family of (square integrable) functions $\left\{\psi^{a ; \epsilon}(t)\right\}$, defined by

$$
\psi^{a ; \epsilon}(t)=\left(\mathcal{V}_{a ; \epsilon} \phi\right)(t)=a \sum_{\ell} g_{\ell}^{(a ; \epsilon)} \phi(a t-\ell)
$$

In the Fourier domain, we have

$$
\widehat{\psi}^{a ; \epsilon}(\omega)=G_{a ; \epsilon}\left(\frac{\omega}{a}\right) \widehat{\phi}\left(\frac{\omega}{a}\right)
$$

where we have set as before

$$
G_{a ; \epsilon}(\theta)=\sum_{k \in \mathbb{Z}} g_{k}^{(a ; \epsilon)} e^{-i k \theta}
$$

However, taking (4.72) into account, we also have for all $a^{\prime} \in \mathcal{A}$

$$
\widehat{\psi}^{a ; \epsilon}(\omega)=G_{a^{\prime} ; \epsilon}\left(\frac{\omega}{a^{\prime}}\right) H_{a^{\prime}}\left(\frac{\omega}{a a^{\prime}}\right) \widehat{\phi}\left(\frac{\omega}{a a^{\prime}}\right)=G_{a^{\prime}, a ; \epsilon}\left(\frac{\omega}{a a^{\prime}}\right) \widehat{\phi}\left(\frac{\omega}{a a^{\prime}}\right)
$$

which reads in the $t$ space

$$
\psi^{a ; \epsilon}(t)=a a^{\prime} \sum_{\ell} g_{\ell}^{\left(a^{\prime}, a ; \epsilon\right)} \phi\left(a a^{\prime} t-\ell\right)
$$

We shall see later on the consequences of this identity on the algorithms for discrete wavelet transform. 

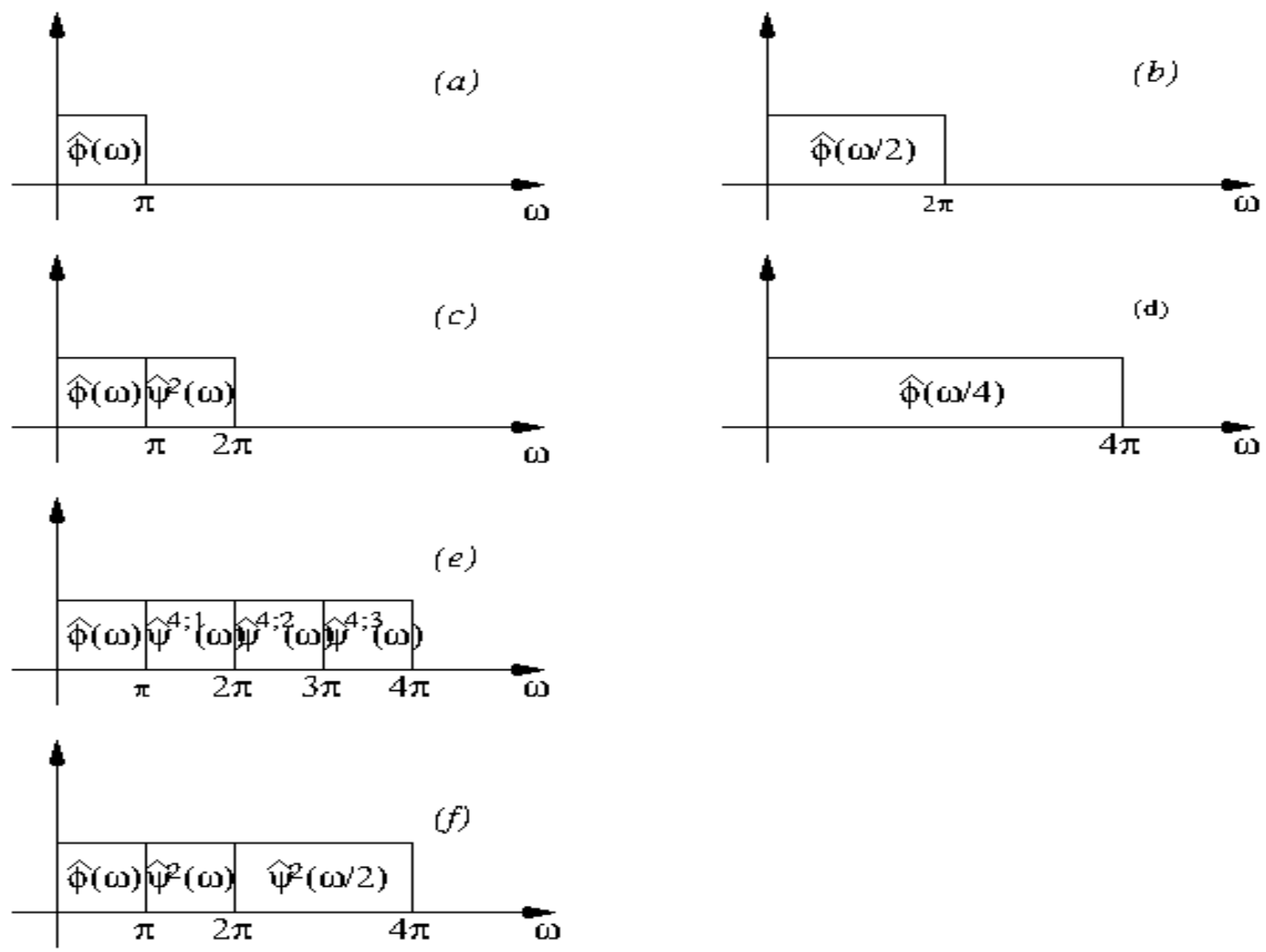

Figure 1: The case of Shannon's MRA, in the Fourier domain. (a): Fourier transform of the scaling function. (b) and (c): two possible partitions of the Fourier domain at scale 1/2. (d), (e) and (f): three possible partitions at scale $1 / 4$. 
The graphs of some of the functions corresponding to the filters described in Example 3 (Shannon's MRA) are given in Fig. 1. There, the Fourier transform of the scaling function $\widehat{\phi}(\omega)$ is the characteristic function of $[-\pi, \pi]$, and the different wavelets $\widehat{\psi}^{a, \epsilon}(\omega)$ are characteristic functions too. This figure illustrates the differences between the different wavelets.

Remark 10 Observe that if one replaces $\phi$ by $D_{a_{0}} \phi, a_{0} \in \mathcal{A}$, in (4.72) and (4.74), then the family of compatible filters $\left\{H_{a}: a \in \mathcal{A}\right\}$ is replaced by its image under the action of $a_{0}$, that is $H$ is replaced by $a_{0} H$. Explicitly,

$$
\widehat{D_{a_{0}} \phi}(a \theta)=H_{a}\left(a_{0} \theta\right) \widehat{D_{a_{0}} \phi}(\theta)=\left(a_{0} H_{a}\right)(\theta) \widehat{D_{a_{0}} \phi}(\theta)
$$

and by $(2.12)$ the discrete filters associated to $\left(a_{0} H_{a}\right)$ are $\Delta_{a_{0}} h^{(a)}$.

\subsection{Discrete wavelet transforms}

We now turn to discretized continuous transforms.

Let us first recall that the continuous wavelet transform $\mathcal{W}_{\psi}$ associated to some analyzing wavelet $\psi$ is defined on $L^{2}(\mathbb{R})$ by

$$
\begin{aligned}
\left(\mathcal{W}_{\psi} f\right)(b, a) & =\left\langle T_{b} D_{a} \psi \mid f\right\rangle \\
& =\frac{1}{\sqrt{|a|}} \int_{\mathbb{R}} \bar{\psi}\left(\frac{x-b}{a}\right) f(x) d x, \quad \forall(b, a) \in \mathbb{R} \times \mathbb{R}^{*}, f \in L^{2}(\mathbb{R})
\end{aligned}
$$

Several approaches to the discretization of such transforms have been proposed (see e.g. [9, $22]$ ). We shall limit ourselves to the multiresolution-type discretization, in which the continuous parameters $a$ and $b$ are replaced with discrete parameters of the form $a_{j}=a_{0}^{j}$ and $b_{j k}=k b_{0} a_{0}^{j}$, where $j$ and $k$ are integers. The situation we face here is slightly different, for we have to consider a scaling function $\phi(t)$ and several wavelets $\psi^{a ; \epsilon}(t)$, associated with different scalings. Given a lattice $\Gamma \subset \mathbb{R}$ and a discrete semigroup $\mathcal{A} \subset \mathbb{Z}_{+}^{*}$, we shall therefore be interested in coefficients of the form

$$
\left(\mathcal{T}_{u ; \epsilon} f\right)(b, a)=\frac{1}{\sqrt{a}} \int \overline{\psi^{u ; \epsilon}}\left(\frac{t-b}{a}\right) f(t) d t,
$$

where $b \in \Gamma$ and $a \in \mathcal{A}$ is any multiple of $u \in \mathcal{A}$, and $\epsilon=1 \ldots d(u)-1$. We are also interested in

$$
(\mathcal{S} f)(b, a)=\frac{1}{\sqrt{a}} \int \bar{\phi}\left(\frac{t-b}{a}\right) f(t) d t,
$$

where $a \in \mathcal{A}$ and $b \in \Gamma$. We shall see how the theory of compatible filters developed in the previous sections provide a direct access to such coefficients.

The connection to the discussion of the previous section is the following. Let $s \in \ell^{2}(\mathbb{Z})$ be any (finite energy) sequence. Equation (4.70) tells us that one can always find $f \in L^{2}(\mathbb{R})$ such that $s_{k}=\int f(t) \bar{\phi}(t-k) d t$. In other words, using the terminology of [17], the sequence

\footnotetext{
${ }^{1}$ That property may also be called self-similarity, since it expresses the fact that the scaling function $\phi$ is invariant under the rescaling $\mathcal{U}_{a}$ for all $a \in \mathcal{A}$.
} 
$s=\left\{s_{k}, k \in \mathbb{Z}\right\}$ may be obtained by sampling imperfectly some $f$ with respect to the lattice $\mathbb{Z}$ and the function $\phi$, that is

$$
s=\Xi_{\mathbb{Z}}(\tilde{\phi} * f)=\Xi_{\mathbb{Z}}^{\phi} f .
$$

Let us consider the Fourier transform of $s$, given by $\widehat{s}(\theta)=\sum_{k \in \mathbb{Z}} s_{k} e^{-i k \theta}$. With the considerations stated in Example 1 the Poisson summation formula (C.106) yields:

$$
\widehat{s}(\theta)=\sum_{\chi \in \mathbb{Z}^{\perp}} \widehat{\tilde{\phi} * f}(\theta+\chi)=\sum_{\ell \in \mathbb{Z}} \widehat{\widehat{\phi}(\theta+2 \pi \ell)} \widehat{f}(\theta+2 \pi \ell)
$$

Let us now set

$$
\begin{aligned}
s_{k}^{a} & =\left(\mathcal{D}_{a}^{*} s\right)_{k} \\
d_{k}^{a ; \epsilon} & =\left(\widetilde{\mathcal{D}}_{a ; \epsilon}^{*} s\right)_{k},
\end{aligned}
$$

and more generally, for $a, u \in \mathcal{A}$, and $\epsilon=1, \ldots d(u)-1$,

$$
d_{k}^{a, u ; \epsilon}=\left(\widetilde{\mathcal{D}}_{a, u ; \epsilon}^{*} s\right)_{k}=\left(\widetilde{\mathcal{D}}_{u ; \epsilon}^{*} \mathcal{D}_{a}^{*} s\right)_{k} .
$$

The last equality is a consequence of Equation (2.30). We notice that the action of the adjoint pseudodilation $\mathcal{D}_{a}^{*}$ in the Fourier domain reads

$$
\widehat{\mathcal{D}_{a}^{*} s}(\theta)=\frac{1}{a} \sum_{\ell=0}^{a-1} \overline{H_{a}}\left(\frac{\theta+2 \pi \ell}{a}\right) \widehat{s}\left(\frac{\theta+2 \pi \ell}{a}\right) .
$$

Then we have

$$
\begin{aligned}
s_{k}^{a} & =\frac{1}{2 \pi} \frac{1}{a} \int_{-\pi}^{\pi} e^{i k \theta} \sum_{\ell=0}^{a-1} \overline{H_{a}}\left(\frac{\theta+2 \pi \ell}{a}\right) \widehat{s}\left(\frac{\theta+2 \pi \ell}{a}\right) d \theta \\
& =\frac{1}{2 \pi} \int e^{i k a \theta} \widehat{f}(\theta) \overline{H_{a}}(\theta) \overline{\widehat{\phi}}(\theta) d \theta \\
& =\frac{1}{2 \pi} \int e^{i k a \theta} f(\theta) \bar{\phi}(a \theta) d \theta .
\end{aligned}
$$

In other words

$$
s_{k}^{a}=\frac{1}{a} \int f(t) \bar{\phi}\left(\frac{t}{a}-k\right) d t=\frac{1}{\sqrt{a}}(\mathcal{S} f)(a k, a) .
$$

Similarly, we obtain

$$
d_{k}^{a ; \epsilon}=\frac{1}{2 \pi} \int_{\mathbb{R}} e^{i a k \theta} \widehat{f}(\theta) \overline{G_{a ; \epsilon}}(\theta) \overline{\widehat{\phi}}(\theta) d \theta=\frac{1}{a} \int f(t) \overline{\psi^{a ; \epsilon}}\left(\frac{t}{a}-k\right) d t=\frac{1}{\sqrt{a}}\left(\mathcal{T}_{a ; \epsilon} f\right)(a k, a) .
$$

More generally, we are interested in computing coefficients of the form given in (4.79). An immediate calculation shows that

$$
\begin{aligned}
\left(\mathcal{T}_{u ; \epsilon} f\right)(a u k, a u) & =\frac{1}{\sqrt{a u}} \int \overline{\psi^{u ; \epsilon}}\left(\frac{t}{a u}-k\right) f(t) d t \\
& =\sqrt{a u} \sum_{\ell} \bar{g}_{\ell}^{(u ; \epsilon)} \int f(t) \bar{\phi}\left(\frac{t}{a}-u k-\ell\right) d t \\
& =\sqrt{a u} \sum_{\ell} \bar{g}_{\ell-u k}^{(u ; \epsilon)} s_{\ell}^{a} \\
& =\sqrt{a u} d_{k}^{u, a ; \epsilon} .
\end{aligned}
$$


However, using (4.85), we also obtain an alternate expression for the coefficients $\left(\mathcal{T}_{u ; \epsilon} f\right)(k a u, a u)$ :

$$
\left(\mathcal{T}_{u ; \epsilon} f\right)(k a u, a u)=\sqrt{a u}\left(\widetilde{\mathcal{D}}_{u ; \epsilon}^{*} s^{a}\right)_{k} .
$$

We have then obtained an equivalence between continuous and discrete wavelet coefficients. Taking into account the relations satisfied by the scaling function $\phi$ and the wavelets $\psi^{a ; \epsilon}$, we obtain

$$
\begin{gathered}
s_{k}^{a}=\mathcal{D}_{a}^{*} s_{k}=\frac{1}{\sqrt{a}}\left\langle T_{a k} D_{a} \phi \mid f\right\rangle \\
d_{k}^{a ; \epsilon}=\widetilde{\mathcal{D}}_{a ; \epsilon}^{*} s_{k}=\frac{1}{\sqrt{a}}\left\langle T_{a k} D_{a} \psi^{a ; \epsilon} \mid f\right\rangle=\frac{1}{\sqrt{a}}\left\langle T_{a k} D_{a} \mathcal{V}_{a ; \epsilon} \phi \mid f\right\rangle .
\end{gathered}
$$

and

$$
d_{k}^{a, u ; \epsilon}=\widetilde{\mathcal{D}}_{a, u ; \epsilon}^{*} s_{k}=\frac{1}{\sqrt{a u}}\left\langle T_{a u k} D_{a u} \psi^{u ; \epsilon} \mid f\right\rangle=\frac{1}{\sqrt{a u}}\left\langle T_{a u k} D_{a u} \mathcal{V}_{u ; \epsilon} \phi \mid f\right\rangle
$$

Therefore, we end up with two equivalent methods for computing the generalized wavelet coefficients $\left(\mathcal{T}_{u} f\right)(b, a, \epsilon)$, via the associated pseudodilation. This makes the connection between compatible filters and continuously defined wavelets.

We summarize the results in the following theorem:

Theorem 2 Let $\left\{\left(H_{a}, G_{a, u ; \epsilon}\right): a, \in \mathcal{A}, u \neq 1, \epsilon=1, \ldots d(u)-1\right\}$ be a family of compatible filters such that there exists a function $\phi \in L^{1}(\mathbb{R}) \cap L^{2}(\mathbb{R})$ such that

$$
\widehat{\phi}(a \theta)=H_{a}(\theta) \widehat{\phi}(\theta), \quad \forall a \in \mathcal{A}, \forall \theta \in \mathbb{R}
$$

Let $\psi^{a ; \epsilon}$ be defined by

$$
\widehat{\psi}^{a ; \epsilon}(a \theta)=G_{a ; \epsilon}(\theta) \widehat{\phi}(\theta), \quad \forall \theta \in \mathbb{R}
$$

Let $\Xi_{\Gamma}$ be the sampling operator acting on functions on $\mathbb{R}$, and $\Xi_{\Gamma}^{\phi}$ be defined as in (4.81). Then for all $a, u \in \mathcal{A}, u \neq 1$, and $\epsilon=1, \ldots d(u)-1$, the following diagram is commutative

$\mathcal{T}$

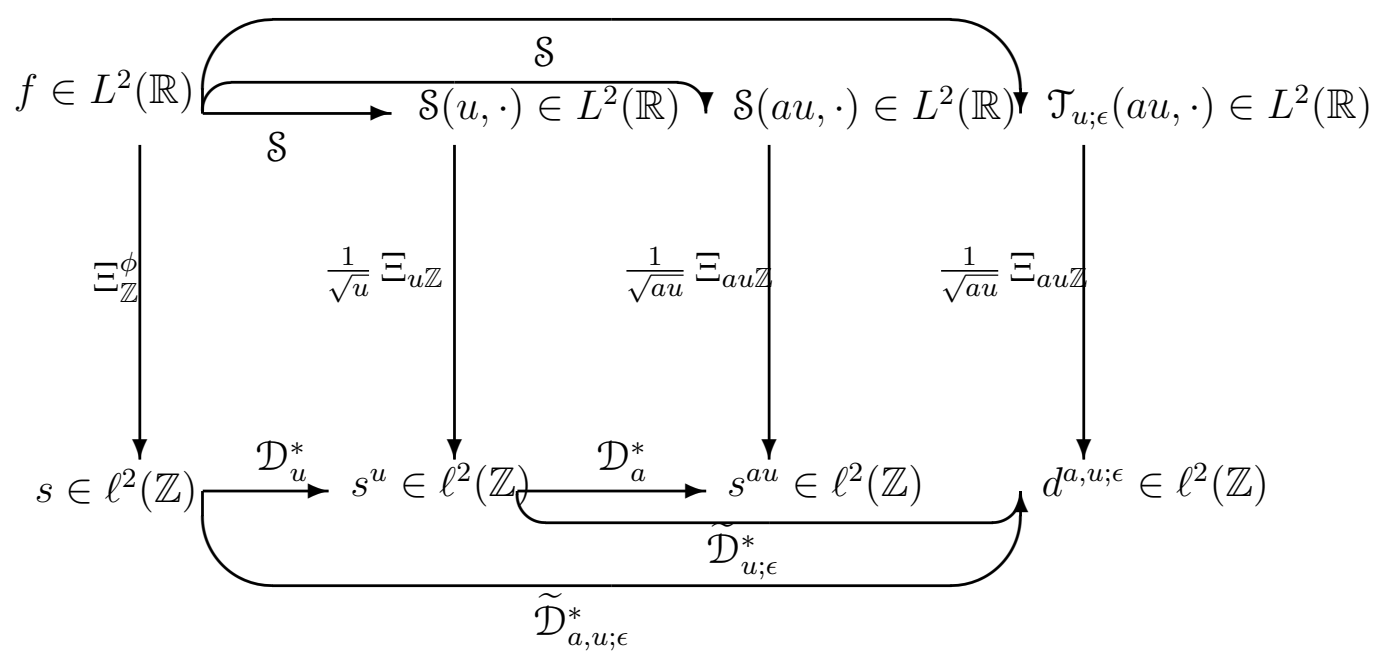


REMARK 11 We notice that the commutative diagram above suggests that the (normalized) sampling operators $\frac{1}{\sqrt{a}} \Xi_{a}$ act as generalized intertwining operators between the representation of the affine group on $L^{2}(\mathbb{R})$ and the representation of the affine semigroup $\mathcal{A} \times \mathbb{Z}$ on $\ell^{2}(\mathbb{Z})$. This may offer a different point of view on the harmonic analysis of the affine group.

REMARK 12 One can check, using the right Haar measure on the full affine group of the line, that the admissibility condition on $\psi$ yields:

$$
\sum_{a \in \mathcal{A}} \sum_{\epsilon=1}^{d(a)-1} \sum_{k \in \mathbb{Z}}\left|d_{k}^{a ; \epsilon}\right|^{2}<\infty
$$

\section{Conclusion}

We have introduced and studied a general setting for multiresolutions associated to the action of a semigroup $\mathcal{A}$ on a lattice $\Gamma$. Special attention has been given to the case where the semigroup is the semidirect product of the group of translations of the lattice with an abelian (dilation) semigroup. The possible actions of such semigroups on $\ell^{2}(\Gamma)$ turn out to be highly constrained. In particular, the (pseudo)dilations are necessarily products of a natural dilation $\Delta_{a}$ by a convolution operator $K_{a} s=s * h^{(a)}$. In addition, the sequences $h^{(a)}, a \in \mathcal{A}$ must satisfy certain compatibility relations. Families of sequences satisfying such compatibility relations are called compatible filters.

The existence of such sequences $h^{(a)}$ turns out to be a difficult problem. Besides the cases covered by classical multiresolution analysis, we only know a few examples of these for bigger semigroups $\mathcal{A}$. We express this existence problem as a cohomology problem, for an appropriate semigroup cohomology. The classification of compatible filters is still an open problem, even in the classical multiresolution case. However, our approach suggests that one may hope to achieve such a classification by means of algebraic topology methods.

The need of constructing families of dilations on sequences which ensure perfect reconstruction of an original sequence from the dilated ones naturally leads to introduce a second family of pseudodilations, called associated pseudodilations. The latter are associated to discrete filters as well, and the corresponding filters also satisfy compatibility relations.

Transporting the action of the pseudodilations to the case of continuously defined functions yields structures which are extremely close to multiresolution analyses. In particular, the pseudodilations provide the algebraic setting for the pyramid algorithms familiar to wavelet specialists. The pyramid algorithm for computing recursively scaling function coefficients is nothing but a recursive use of a pseudodilation (more precisely the adjoint of a pseudodilation). The corresponding wavelet coefficients are obtained via the (adjoint) associated pseudodilations.

Let us close this paper by a few speculative remarks. Even though the proofs of many of the results presented in this paper owe a lot to the classical results of multiresolution analysis, we believe that the approach we developed is original in many respects. First, it expresses a general algebraic setting which covers most of the known multiresolution theories, and as such could offer interesting perspectives for generalizations. Second, it makes a connection with the group theoretical approach to wavelets, which was up to now essentially limited to continuous wavelet transforms. Third, it identifies the problem of existence of filters with a problem 
of cohomological nature, which could provide new hints for the problem of classification of quadrature mirror filters.

Also, we believe that the approach developed here is a first step towards an algebraic formulation of multiresolution analysis, in which the corresponding wavelets could be realized from some semigroup representation, possessing some square-summability property.

\section{Acknowledgements}

Part of this work was done during several visits of the authors to the Institut de Physique Théorique, Université Catholique de Louvain, the Centre de Physique Théorique, CNRS Marseille, and the Laboratoire d'Analyse, Topologie et Probabilités, Université de Provence, Marseille. Those institutes should be thanked for their hospitality.

YBK is supported by the Administration pour la Coopération au Développement (AGCD, Belgium).

\section{References}

[1] S. T. Ali, J.-P. Antoine, J.-P. Gazeau, and U. A. Müller (1995). Coherent states and their generalizations: an overview. Rev. Math. Phys. 7, 1013-1104.

[2] E. Ascher (1972). Extensions et cohomologie de groupes, Lecture notes, Enseignement du troisième cycle de la physique en Suisse Romande (CICP).

[3] M. Baake (1984). Structure and representations of the hyperoctahedral group. J. Math. Phys., 25

[4] D. Bernier and M. Taylor (1996). Wavelets and square integrable representations. SIAM J. Math. Anal. 27, 594-608

[5] C. Bernuau (1998). Wavelet bases associated to a self similar quasicrystal. J. Math. Phys. 39, 4213-4225.

[6] B. Brainerd and R. E. Edwards (1966). Linear operators which commutes with translations. I. Representation theorems. J. Austral. Math. Soc. VI, 289-327.

[7] R. Carmona, W. L. Hwang and B. Torrésani (1998). Practical Time-Frequency Analysis: Wavelet and Gabor Transforms, with an Implementation in S. Academic Press, San Diego.

[8] C.K. Chui (1992). An Introduction to Wavelets. Academic Press, San Diego.

[9] I. Daubechies (1992). Ten Lectures on Wavelets. SIAM, Philadelphia.

[10] K. Flornes, A. Grossmann, M. Holschneider and B. Torrésani (1994). Wavelets on discrete fields. Applied Comput. Harmon. Anal. 1, 134-146.

[11] H. Führ (1996). Wavelet frames and admissibility in higher dimensions. J. Math. Phys. 37, 6353-6366. 
[12] J.-P. Gazeau, J. Patera and E. Pelantová (1998). Tau-wavelets in the plane. J. Math. Phys. 39, 4201-4212.

[13] E. Hernández and G. Weiss (1996). A First Course on Wavelets. CRC Press, Boca Raton, FL.

[14] G. B. Folland (1995). A Course in Abstract Harmonic Analysis. CRC Press, Boca Raton, FL.

[15] K. Gröchenig (1998). Gabor analysis over LCA groups, in Gabor Analysis and Algorithms, H. G. Feichtinger and Th. Strohmer (eds.), Birkhäuser, Basel and Boston, pp. 211-231.

[16] J. R. Higgins (1985). Five short stories about the cardinal series. Bull. Amer. Math. Soc. 12, 45-89.

[17] M. Holschneider (1995). Wavelet analysis over abelian groups. Applied Comput. Harmon. Anal. 2, 52-60.

[18] M. Holschneider (1995). Wavelets: an Analysis Tool. Oxford Univ. Press, Oxford.

[19] M. Holschneider, R. Kronland-Martinet, J. Morlet, and Ph. Tchamitchian (1987). A real-time algorithm for signal analysis with the help of wavelet transform, in Wavelets, Time-Frequency Methods and Phase Space, J.M. Combes, A. Grossmann, and Ph. Tchamitchian (eds.), Springer-Verlag, Berlin, pp. 286-297.

[20] C. P. Johnston (1997). On the pseudodilation representations of Flornes, Grossmann, Holschneider, and Torrésani. J. Fourier Anal. Appl. , 3, 377-385.

[21] S. Lang (1997). Algebra, Addison-Wesley,Reading, MA.

[22] S. Mallat (1998). A Wavelet Tour of Signal Processing. Academic Press, New York, N.Y.

[23] W. Rudin (1962). Fourier Analysis on Groups. Interscience Publ., New York ??

[24] M. Shensa (1993). Wedding the "à trous" and Mallat algorithms. IEEE Trans. Inf. Th. 40, 2464-2482.

[25] G. Steidl (1994). Spline wavelets over $\mathbb{R}, \mathbb{Z}, \mathbb{R} / N \mathbb{Z}$ and $\mathbb{Z} / N \mathbb{Z}$, in Wavelets, Theory, Algorithms and Applications, C. K. Chui, L. Montefusco and L. Puccio (eds.), Academic Press, San Diego.

[26] B. Torrésani (ed.) (1998). Special issue on Wavelets and Time-Frequency Analysis, Journal of Mathematical Physics 39, August 1998.

[27] M. Vetterli and J. Kovacevic (1996). Wavelets and Sub-Band Coding, Prentice Hall, Englewood Cliffs, NJ.

[28] M. V. Wickerhauser (1994). Adapted Wavelet Analysis, from Theory to Software. A.K. Peters, Wellesley, MA. 


\section{Appendix: Background Material, Notations}

We give in this appendix a summary of some basic concepts and useful results on locally compact abelian ( $L C A$ for short) groups. We assume that the fundamental results about Fourier transform and Pontrjagin duality on LCA groups are known. However, the reader not familiar with these results can refer to [23] or any textbook on harmonic analysis (e.g. [14]). Given a LCA group $\mathcal{G}$ (whose group operation shall be written additively), we denote generically by $\mu_{\mathcal{G}}$ the Haar measure on $\mathcal{G}$. When $\mathcal{G}$ is discrete $\mu_{\mathcal{G}}$ is naturally the counting measure and the discrete versions of the relations that we have defined on the whole group $\mathcal{G}$ in a continuous formulation hold again.

\section{A . Notions of harmonic analysis on locally compact abelian groups}

Let $\mathcal{G}$ be a locally compact abelian group with Haar measure $\mu=\mu_{\mathcal{G}}$, and let $\widehat{\mathcal{G}}$ be its unitary dual, that is the set of all unitary characters of $\mathcal{G}$. We use the notation

$$
\omega(x)=\langle\omega, x\rangle \quad(x \in \mathcal{G}, \omega \in \widehat{\mathcal{G}})
$$

to express the duality pairing between $\mathcal{G}$ and $\widehat{\mathcal{G}}$. We denote additively the group law on $\widehat{\mathcal{G}}$ : for any $\chi, \chi^{\prime} \in \widehat{\mathcal{G}}$, we have $\left\langle\chi+\chi^{\prime}, x\right\rangle=\langle\chi, x\rangle\left\langle\chi^{\prime}, x\right\rangle, x \in \mathcal{G}$.

The Fourier transform is defined as usual: given $f \in L^{1}(\mathcal{G})$, its Fourier transform is the function $\mathcal{F} f=\widehat{f} \in L^{\infty}(\widehat{\mathcal{G}})$ defined by

$$
\widehat{f}(\omega)=\int_{\mathcal{G}} f(x) \overline{\langle\omega, x\rangle} d \mu(x) .
$$

It is a standard result (see e.g. [23] for more details) that the so-defined Fourier transform extends to an isometry between $L^{2}(\mathcal{G})$ and $L^{2}(\widehat{\mathcal{G}})$. More precisely, there exists a measure $\mu_{\widehat{\mathcal{G}}}$ on $\widehat{\mathcal{G}}$ (the dual Haar measure) such that the following Plancherel formula holds: for all $f \in L^{2}(\mathcal{G}), \widehat{f} \in L^{2}(\widehat{\mathcal{G}})$ and

$$
\int_{\widehat{\mathcal{G}}}|\widehat{f}(\omega)|^{2} \mu_{\widehat{\mathcal{G}}}(\omega)=\int_{\mathcal{G}}|f(x)|^{2} d \mu(x) .
$$

Let $\Gamma \subset \mathcal{G}$ be any subgroup of $\mathcal{G}$, and let $\Gamma^{\perp} \subset \widehat{\mathcal{G}}$ be its annihilator, defined as

$$
\Gamma^{\perp}=\{\omega \in \widehat{\mathcal{G}}:\langle\omega, x\rangle=1 \text { for all } x \in \Gamma\}
$$

$\Gamma^{\perp}$ is a closed subgroup of $\widehat{\mathcal{G}}$. We always have $\Gamma \subset\left(\Gamma^{\perp}\right)^{\perp}$ and the reverse inclusion is true if $\Gamma$ is itself a closed subgroup of $\mathcal{G}$.

There is a neat duality between subgroups and quotient groups of a locally compact abelian group as stated in the following theorem; the proof uses the Pontrjagin duality theorem and is given in [23, Theorem 2.1.2] and also in [14, Theorem 4.39]. 
Theorem A.1 Let $\Gamma$ be a closed subgroup of $\mathcal{G}$ and define

$$
\Phi: \widehat{\mathcal{G} / \Gamma} \rightarrow \Gamma^{\perp} \quad \text { and } \quad \Psi: \widehat{\mathcal{G}} / \Gamma^{\perp} \rightarrow \widehat{\Gamma}
$$

by $\Phi(\eta)=\eta \circ q, \quad \Psi\left(\omega \Gamma^{\perp}\right)=\left.\omega\right|_{\Gamma}$, where $q: \mathcal{G} \longrightarrow \mathcal{G} / \Gamma$ is the canonical projection. Then $\Phi$ and $\Psi$ are isomorphisms of topological groups.

Note that the quotient groups $\mathcal{G} / \Gamma$ and $\widehat{\mathcal{G}} / \Gamma^{\perp}$ are both locally compact abelian groups. The main idea of Theorem A.1 is that, on the one hand, the characters of $\mathcal{G} / \Gamma$ can be identified with the subset $\Gamma^{\perp}$ of $\widehat{\mathcal{G}}$ and, on the other hand, $\widehat{\Gamma}$ can be identified with the quotient $\widehat{\mathcal{G}} / \Gamma^{\perp}$. Note also that the surjectivity of $\Psi$ yields a sort of Hahn-Banach theorem for locally compact abelian groups, in the sense that every character on $\Gamma$ extends to a character of $\mathcal{G}$.

Let us now suppose that $\Gamma$ is a lattice in $\mathcal{G}$, that is $\Gamma$ is a discrete subgroup of $\mathcal{G}$ and the quotient $\mathcal{G} / \Gamma$ is compact. By a fundamental domain of $\Gamma$ in $\mathcal{G}$, we mean a $\mu$-measurable set $\Omega \subset \mathcal{G}$ such that for each $x \in \mathcal{G}, \Omega \cap(x+\Gamma)$ consists of a single point. An equivalent definition is given in [15]. Given a lattice $\Gamma \subset \mathcal{G}$, it can be shown that there always exists a fundamental domain $\Omega$ of $\Gamma$ in $\mathcal{G}$. It is standard to take $\Omega=\mathcal{G} / \Gamma$, but many other choices are possible (see for instance [16] for various choices of fundamental domains). The lattice size, which we denote by $s(\Gamma)$, is defined as the measure of a fundamental domain, that is, $s(\Gamma)=\mu(\Omega)$, and it is independent of the particular choice of $\Omega$. The quantity $s(\Gamma)^{-1}$ then serves as a measure of the density of $\Gamma$. Taking $\Omega=\mathcal{G} / \Gamma$ allows us to take the measure naturally inherited from $\mathcal{G}$ as the measure of $\mathcal{G} / \Gamma$; we normalize it to be a probability measure such that the Weyl formula (see e.g. [14] for details) holds: for all $f \in L^{1}(\mathcal{G})$,

$$
\int_{\mathcal{G}} f(x) d \mu(x)=\int_{\mathcal{G} / \Gamma} \sum_{\gamma \in \Gamma} f(x+\gamma) d \mu_{\mathcal{G} / \Gamma}(x) .
$$

If $\Gamma$ is a lattice in $\mathcal{G}$, then its annihilator $\Gamma^{\perp}$ is a lattice in $\widehat{\mathcal{G}}$ and $s(\Gamma) s\left(\Gamma^{\perp}\right)=1$ (see, for instance, [15, Lemma 6.2.3]). If $\Gamma_{i}(i=1,2)$ are two lattices in $\mathcal{G}$, such that $\Gamma_{2} \subset \Gamma_{1}$, then in the dual space we have $\Gamma_{1}^{\perp} \subset \Gamma_{2}^{\perp}$; furthermore, in all practical situations we know, it is always possible to choose a fundamental domain $\Omega_{i}$ of $\Gamma_{i}, i=1,2$, in such a way that, as subsets of $\mathcal{G}$, they satisfy $\Omega_{1} \subset \Omega_{2}$ (see for example $[16]$ ).

The whole discussion on the existence and choices of fundamental domains may be repeated in the dual space. In particular, we have the following version of the Weyl formula: for all $f \in L^{1}(\widehat{\mathcal{G}})$,

$$
\int_{\widehat{\mathcal{G}}} f(\chi) d \mu_{\widehat{\mathcal{S}}}(\chi)=\int_{\widehat{\widehat{\mathcal{S}}} / \Gamma^{\perp}} \sum_{\lambda \in \Gamma^{\perp}} f(\chi+\lambda) d \mu_{\mathcal{G} / \Gamma^{\perp}}(\chi) .
$$

If $\Gamma_{2} \subset \Gamma_{1} \subset \mathcal{G}$ are two nested lattices in $\mathcal{G}$ such that the quotient group $Q=\Gamma_{1} / \Gamma_{2}$ is finite of order $d=|Q|$, then it is always possible to choose fundamental domains $\Omega_{1}$ and $\Omega_{2}$ for $\Gamma_{1}^{\perp}$ and $\Gamma_{2}^{\perp}$ in $\widehat{\mathcal{G}}$ such that $\Omega_{2} \subset \Omega_{1}$, and

$$
\Omega_{1}=\bigcup_{\epsilon=0}^{d-1}\left(\Omega_{2}+\chi_{\epsilon}\right),
$$

where $\chi_{\epsilon} \in \widehat{\mathcal{G}}, \epsilon=0, \ldots d-1$, and the sets $\Omega_{2}+\chi_{\epsilon}$ intersect each other at most on a $\mu_{\widehat{\mathcal{G}}^{-}}$ negligible set. 


\section{B . Basic operations}

The basic operators are introduced in the following way.

The translation of parameter $b \in \mathcal{G}$ is the unitary operator $T_{b}$ defined on $L^{2}(\mathcal{G})$ by

$$
\left(T_{b} f\right)(x)=f(x-b) .
$$

The modulation of parameter $b \in \mathcal{G}$ is the unitary operator $E_{b}$ defined on $L^{2}(\widehat{\mathcal{G}})$ by

$$
\left(E_{b} \widehat{f}\right)(\omega)=\overline{\langle\omega, b\rangle} \widehat{f}(\omega)
$$

The maps $b \stackrel{T}{\longmapsto} T_{b}$ and $b \stackrel{E}{\longmapsto} E_{b}$ provide two unitarily equivalent representations of $\mathcal{G}$ (with respective carrier spaces $L^{2}(\mathcal{G})$ and $\left.L^{2}(\widehat{\mathcal{G}})\right)$ and the associated interwining operator is the Fourier transform. Explicitly we have

$$
\mathcal{F} T_{b}=E_{b} \mathcal{F} \text { for all } b \in \mathcal{G} \text {. }
$$

If $f$ and $g$ are two functions over $\mathcal{G}$, their convolution product is defined as

$$
(f * g)(x)=\int_{\mathcal{G}} f(x-y) g(y) d \mu(y),
$$

if the integral in the r.h.s. converges. The convolution product is associative and since $\mathcal{G}$ is abelian, it is also commutative, that is,

$$
(f * g) * h=f *(g * h), \quad f * g=g * f .
$$

In the Fourier space, the convolution is given by pointwise multiplication:

$$
\widehat{f * g}=\widehat{f} \widehat{g} .
$$

\section{C . Sampling}

A natural map from functions defined over the whole group $\mathcal{G}$ to functions over the lattice $\Gamma$ is given by the sampling operator (perfect sampling in the terminology of [18]) $\Xi_{\Gamma}$ associated to $\Gamma$, defined as

$$
\Xi_{\Gamma} f=f_{\left.\right|_{\Gamma}}
$$

where $f$ is a function with suitable properties (i.e. for which point values make sense).

A function $f$ over $\mathcal{G}$ is said to be $\Gamma$-periodic if for all $x \in \mathcal{G}$,

$$
f(x+\gamma)=f(x) \text {, for all } \gamma \in \Gamma .
$$

Such a function can be identified with a function over $\mathcal{G} / \Gamma$ in the usual way.

The periodization operator is defined as

$$
P: L^{1}(\mathcal{G}) \rightarrow L^{1}(\mathcal{G} / \Gamma), \quad(P f)(x)=\sum_{\gamma \in \Gamma} f(x+\gamma) .
$$


By the Weyl formula (see e.g. [14, Eq. (2.50)]), we have

$$
\langle\phi \mid f\rangle_{L^{2}(\mathcal{G})}=\langle\phi \mid P f\rangle_{L^{2}(\mathcal{G} / \Gamma)} \text {, for any } \Gamma \text {-periodic function } \phi \text {. }
$$

In other words, the periodization operator allows us to write the scalar product of a $\Gamma$-periodic function $\phi$ with an arbitrary function $f$ over $\mathcal{G}$ as a scalar product over $\mathcal{G} / \Gamma$. In the particular case where $\mathcal{G}=\mathbb{R}$ and $\Gamma=\mathbb{Z}$, the Fourier transform of a sequence is a $\mathbb{Z}^{\perp}$-periodic function, i.e a $2 \pi$-periodic function.

We close this section by giving the useful Poisson Summation formula, which links sampling to periodization via the Fourier transform. The proof can be found in most treatises on harmonic analysis (see for instance [14, Theorem 4.42], [15, Lemma 6.2.2], and also [18]) .

TheOREM C.1 (Poisson Summation Formula) Let $\Gamma \subset \mathcal{G}$ be a lattice. If $f \in L^{1}(\mathcal{G}) \cap \mathcal{F}^{-1} L^{1}(\widehat{\mathcal{G}})$, then

i) The $\Gamma$-periodized $\phi(\dot{x})=\sum_{\gamma \in \Gamma} f(x+\gamma)$ of $f$ is in $L^{1}(\mathcal{G} / \Gamma)$ (where $\dot{x}=x+\Gamma$ ) and for $\eta \in \widehat{\mathcal{G} / \Gamma} \simeq \Gamma^{\perp}$

$$
\widehat{\phi}(\eta)=\frac{1}{s(\Gamma)} \widehat{f}(\eta)
$$

ii) We have

$$
\begin{array}{ll}
\sum_{\gamma \in \Gamma} f(x+\gamma) & =\frac{1}{s(\Gamma)} \sum_{\eta \in \Gamma^{\perp}} \widehat{f}(\eta)\langle\eta, x\rangle \quad \text { a. e. } \\
\sum_{\chi \in \Gamma^{\perp}} \widehat{f}(\omega+\chi) & =s(\Gamma) \sum_{x \in \Gamma} f(x) \overline{\langle\omega, x\rangle} \quad \text { a. e. }
\end{array}
$$

iii) If, in addition, $\sum_{\eta \in \Gamma^{\perp}}|\widehat{f}(\eta)|^{2}<\infty$, then $\phi \in L^{2}(\mathcal{G} / \Gamma)$ and the relations (C.105) and (C.106) hold in the $L^{2}$ - sense. 